\title{
Early and Late Selection in Unconscious Information Processing
}

\author{
Carsten Pohl and Andrea Kiesel \\ University of Würzburg
}

\author{
Wilfried Kunde \\ University of Dortmund
}

\author{
Joachim Hoffmann \\ University of Würzburg
}

\begin{abstract}
In four experiments, we investigated whether masked stimuli in priming experiments are subjected to early or to late selection. In Experiment 1, participants classified four target-pictures as being small or large. In line with early selection accounts, prime-pictures with a different perceptual appearance as the experienced targets did not elicit congruency effect. In Experiment 2, 40 targets all depicting animals were presented. Results were in line with late selections assumptions because novel animal primes but not novel primes form different semantic categories yielded congruency effects. In Experiment 3, the targets were chosen such that there is a second semantic feature that covaried with the required response. Here, novel primes picturing small animals did not influence target responses with regard to the instructed size classification, but with regard to their affiliation to the category animal.In Experiment 4, small and large pictures from two categories were presented. Category match did not influence priming, ruling out that feature overlap contaminated the former results. The results indicate that participants' prestimulus expectations determine in which stage in the processing-stream masked stimuli are selected.
\end{abstract}

Keywords: early/late selection, masked priming

Perception is basically selective (cf. Müller \& Krummenacher, 2002; Pashler, 1999). At every moment, we perceive only a fraction of the millions of stimuli hitting on our senses. For example, besides reading these lines you can see a multitude of letters on the paper, you might also see parts of your computer and desk, you might hear noise from inside or outside your room and so on. Nevertheless, only a fraction of this information is perceived at the same time. At the moment you probably perceive no more than exactly the words you are currently reading.

The issue which stimuli are selected for perception (i.e. conscious identification) engages researchers since decades (Cherry, 1953; Corteen \& Dunn, 1974; Corteen \& Wood, 1972; Dawson \& Schell, 1982; Egeth, 1977; Moray, 1959; Ostry, Moray, \& Marks, 1976; Treisman, 1964). Obviously, selection presupposes that there is a vast amount of information of which parts can be chosen. Accordingly, it is assumed that stimuli initially and preattentively activate representations, with some of them processed further while others get lost. In this framework it is debated

Carsten Pohl, Andrea Kiesel, and Joachim Hoffmann, Department of Psychology, University of Würzburg; and Wilfried Kunde, Department of Psychology, University of Dortmund.

This research was funded through Deutsche Forschungsgemeinschaft Grants HO 1301/10-1, KI 1388/1-2 and Ku 1964/1-1,2 warded to Joachim Hoffmann, Andrea Kiesel, and Wilfried Kunde.

We thank Bernhard Hommel, Tony Greenwald, and two anonymous reviewers for helpful comments on an earlier version of the article. Further, we thank Alexander Heinemann for making the result graphs.

Correspondence concerning this article should be addressed to Carsten Pohl, University of Würzburg, Institut für Psychologie III, 97070 Würzburg, Germany. E-mail: pohl@psychologie.uni-wuerzburg.de or Andrea Kiesel (kiesel@psychologie.uni-wuerzburg.de). since long whether the pre-attentively activated representations are either restricted to elementary perceptual features like location, color, size, shape, pitch, etc. or whether these preattentive representation comprise the conceptual meaning of the registered stimuli.

Broadbent suggested "early selection" mechanisms by claiming that "... the operation taking the form of selecting information from all sensory events having some features in common" (1958, p. 297). On the other hand, Deutsch and Deutsch held a "late selection" view that "... all sensory messages which impinge the organism are perceptually analyzed at the highest level" (1963, p. 85).

This controversy between early and late selection has been debated passionately. Empirical evidence more in line either with the one or the other position was brought forward. Presumably early and late selection positions do not exclude each other. Rather concrete experimental conditions determine whether selection takes place on the basis of elementary features or on the basis of the meaning of the stimuli.

The locus of selection debate was restricted to supraliminally presented stimuli. Thereby, processing of stimuli beyond the selection stage was equated with conscious perception, that is, with becoming aware of them. Interestingly, in the last years the debate on early or late selection has been revived with respect to a new paradigm - priming by subliminal stimuli. In a typical subliminal priming experiment, participants perform a speeded two-choice reaction time task; for example, they indicate whether a presented target word denotes an object that is small or large (Damian, 2001). The target is preceded by a prime stimulus, presented very briefly and between masks to prevent conscious awareness. When prime and target afford the same response (congruent trial) rather than different responses (incongruent trial) responding is faster and more accurate. This so-called priming or congruency effect 
emerges as a consequence of unconscious prime processing. It indicates that the prime is processed to such a degree that it influences performance to a subsequently presented target.

Priming by subliminal stimuli has become a well-established method to investigate the phenomenon of unconscious stimulus processing, and it has been observed in many recent studies (e.g. Ansorge \& Neumann, 2005; Damian, 2001; Dehaene et al., 1998; Greenwald, Draine, \& Abrams, 1996; Kunde, Kiesel, \& Hoffmann, 2003). Following this basic finding, the question arises upon which features subliminally presented prime stimuli evolve their impact on responding to the target. At a first sight, it seems contradictory to extent the question of early versus late selection to stimuli that remain unconscious because one may argue that the fact that these stimuli remain unconscious indicates that the stimuli do not become selected at all. Yet, when assuming that selection does not occur in order to restrict conscious representation of the outer world but that selection occurs as a mechanism of action control ("selection for action," Allport, 1987), the question whether subliminal stimuli are subject to early or late selection becomes meaningful.

On the one hand, there is empirical evidence indicating that only elemental features of the subliminal stimuli are processed and elicit congruency effects. For example, in an affective evaluation task by Abrams and Greenwald (2000), target words were categorized according to their meaning as pleasant or unpleasant. Prime stimuli resembled the targets because of similar letter chains. Importantly, physical similarity and not the affective connotation of the prime words determined the resulting congruency effects. For example, contrary to its pleasant connotation the prime smile facilitated responding to unpleasant targets after participants had repeatedly classified the unpleasant words smut and bile. Within the same line, Greenwald, Abrams, Naccache, and Dehaene (2003) let participants classify two-digit numbers as smaller or larger than 55. All targets requiring the one or the other response were constructed from a pool of four single figures, respectively (e.g. $16,49 ; 73,82)$. After a training phase with these targets, primes were presented whose single figures derived from the set of the opposite target set (e.g. 64, 91; 27, 38). Paradoxically, these primes had no impact on the targets according to their two-digit number value but according to their single digits which were associated with targets from the opposite response. Both studies demonstrated that primes were not processed regarding their meaning as a whole. On the contrary, primes elicited congruency effects according to parts of them which were associated with a certain response. Taken together, these observations suggest that masked stimuli are not inevitably processed semantically but that unconscious processing is limited to elemental features ("sensory events") as Broadbent (1958) claimed in his early selection account.

On the other hand, it has been claimed that also "nonconscious perceptual processes automatically redescribe sensory data into every representational form and to the highest levels of description available to the organism" (Marcel, 1983, p. 238). Although the underlying studies were initially rejected mostly due to methodologically reasons (cf. Holender, 1986), there is accumulating new evidence, suggesting that primes are able to influence target responses via their semantics or meaning. For example, Abrams, Klinger, and Greenwald (2002) found that prime words elicited congruency effects according to their semantic category and not due to learned S-R links. After having practiced unpleasant and pleasant words with a left and a right hand response, key assignments were reversed and the former practiced words served now as primes. These primes still influenced responses to new targets with their actual valence although the practiced mapping would have demanded the contrary response. Semantic congruency effects were also reported for a number categorization task (Dehaene et al., 1998). As targets, Arabic digits as well as number words were used. Priming remained significant for primes which were presented in another notation as the targets and when identical repetitions of prime and target were removed. Dehaene and colleges concluded "that the prime was unconsciously processed according to task instructions, all the way down to the motor system" (1998, p. 597). Furthermore, in a study by Dell'Acqua and Grainger (1999), semantic activation spread even from picture primes to word targets. Participants were asked to decide whether 1 of 84 different words was denoting an artificial or natural thing. Line drawings of these 84 different concepts were presented as primes. Facilitation for the target responses was observed when the target was preceded by a prime from the same semantic category (artificial or natural). Thus, the meaning that was necessary for the classification task must have been processed from the prime pictures. In addition to behavioral data, there is also evidence for automatic spreading activation of masked primes from eventrelated brain potentials. In a lexical decision task, semantic relatedness between prime words and target words modulated the N400, which reflects semantic processing (Kiefer, 2002; Kiefer 2007; Kiefer \& Spitzer, 2000). To sum up, these observations imply in accordance with the late selection account of Deutsch and Deutsch that unconscious stimuli "are perceptually analysed at the highest level" (Deutsch \& Deutsch, 1963, p. 85).

Based on these findings, we intend to demonstrate in the present study that similar conclusions that have been derived from the selection of supraliminal stimulation apply to the selection of subliminal stimulation as well. Early as well as late selection might occur. In a recent priming study, Klauer, Eder, Greenwald, and Abrams (2006; Exp. 3) already observed that varying prime duration leads to early or late selection of subliminal primes. Participants' task was to classify one of 24 target words according to their gender. When presented for a short time $(28 \mathrm{~ms})$, only target primes but not novel prime were effective. For longer prime durations (42 ms), however, even novel primes elicited congruency effects, which suggests that semantic processing might take a bit more time to unfold than perceptual processing.

Our basic approach in the present study was to demonstrate that essentially the same subliminal stimulus input (inclusive equal prime duration) can either affect responding through perceptual features or through conceptual meaning depending on concrete experimental conditions. Whether the same unconscious stimuli affect responses according to their "early" perceptual features or their "late" conceptual meaning depends on the perceivers/actors intentions, which are shaped by the consciously experienced task affordances.

\section{Overview of the Experiments}

The first two experiments were designed to find evidence for early and late selection of masked stimuli, respectively. In both experiments, participants had to classify animals as being small or large. In Experiment 1, we presented only four different animal 
pictures as targets so that participants could perform the task just based on the mere sensory input. As a consequence, only the very same prime pictures as the target pictures elicited congruency effects; all other novel primes remained ineffective. Even pictures depicting the same animals but in a different illustration with reversed left-right orientation did not induce a congruency effect, demonstrating early selection of masked stimuli. In Experiment 2, we enlarged the target set to 40 animal pictures so that it was necessary to consider the real physical size of the target animals. Priming transferred to novel primes, but was restricted to novel primes from the same category as the experienced targets (animals). Pictures belonging to other semantic categories (inanimate objects) failed to evoke congruency effects despite they could have been easily classified as small or large. Thus, these results are in line with late selection of masked stimuli.

Experiment 3 further elaborated on possible determinants of late selection settings. Is it possible that selection becomes driven by a semantic but not instructed semantic categorization? In Experiment 3, the task again required a small/large-classification of a large target set of 40 pictures. All pictures requiring a small response depicted objects, and all pictures requiring a large response depicted animals. Again, priming transferred to novel primes; but contrary to the originally instructed classification, prime pictures of small animals primed the response that was required for the category "large." Hence, these primes elicited paradoxical congruency effects opposite to their instructed meaning. We therefore conjecture that the initially instructed large response got reinterpreted as an animal response.

Finally, in Experiment 4 we wanted to find out whether overlapping perceptual features between prime and target pictures in our experiments had impact on priming. Therefore, we presented again a large target set of 40 pictures now consisting of 10 pictures of small animals, small objects, large animals, and large objects, respectively, and asked participants to perform a small/largeclassification. Thus, in contrast to Experiment 3 the animal-object categories were nonpredictive for the required response. The observed congruency effects did not depend on whether primes and targets belonged to the same animal/object category. Moreover, the extent of feature overlap had no significant impact on responding, ruling out that feature overlap contaminated the former results.

\section{Experiment 1}

The purpose of Experiment 1 was to examine whether attention can operate as an early filter in the stream of processing for masked stimuli. Does priming transfer to novel prime stimuli when using a small target set with targets from a semantically related category, like animals? Or is priming confined to the few target stimuli, especially when they can be discriminated merely on the basis of sensory features?

Participants were instructed to categorize depicted animals as small or large. Target stimuli were four grey shaded animal pictures. Four different types of primes were used: pictures of animals that were also presented as target pictures (target primes, TP), pictures of animals that were presented in a different illustration as the targets, i.e. as black line drawings (different illustration target primes, Diff-Illu-TP), pictures of animals that were never presented as targets (same category novel primes, Same-Cat-NP), and pictures of objects (different category novel primes, Diff-Cat-NP).
These four different types of primes allow us to assess systematically how early selection of primes sets in. A very early filter would let pass just sensory identical stimuli, which would be indicated by priming confined to TPs. Congruency effects spreading to pictures of the same animals but presented in a different illustration (Diff-Illu-TPs) would demonstrate a somewhat later selection, whereas congruency effects of all animal pictures (Same-Cat-NP) would reveal late selection. Finally, when even pictures of objects belonging to a different category would elicit priming, then no filter at all would operate resulting in latest selection, that is, complete semantic processing of all stimuli. As the target set in Experiment 1 is limited to only four different animal pictures it should be advantageous to select the response on the basis of stimuli's sensory features. Thus, we expect that Experiment 1 provides a favorable condition for early selection of masked stimuli.

\section{Method}

\section{Participants}

Twenty-four students (seven males) of the University of Würzburg with an average age of 23.0 years participated in Experiment 1 in fulfillment of course requirements or payment. All reported having normal or corrected-to-normal vision, and were not familiar with the purpose of the experiment. An experimental session lasted approximately $45 \mathrm{~min}$.

\section{Apparatus and Stimuli}

An IBM-compatible computer with a 17-inch VGA-Display and the software package E-Prime (Schneider, Eschman, \& Zuccolotto, 2002) were used for stimulus presentation and response sampling. Stimulus presentation was synchronized with the vertical retraces of a $70-\mathrm{Hz}$ monitor, resulting in a refresh rate of $14.3 \mathrm{~ms}$. Responses were executed with the index fingers of both hands and collected with an external keyboard with three response keys (1.7 $\mathrm{cm}$ width, distance $0.2 \mathrm{~cm}$ ); the middle response key was not used.

The pictures used as primes and targets were derived from the grey scale-shaded images set of "Snodgrass and Vanderwart-like" objects (Rossion \& Pourtois, 2004; see http://alpha.cog.brown.edu: 8200/stimuli/objects/svlo.zip/view) and the black line drawings from the International Picture-Naming Project (e.g. Bates et al., 2003; see http://crl.ucsd.edu/ aszekely/ipnp/). ${ }^{1}$ The target set consisted of four pictures of animals (lion, zebra, mouse, and snail) that could be easily classified as being smaller or larger than the reference object (a box, measuring $51 \times 50 \times 24 \mathrm{~cm}$ ). Primes were 16 pictures: 4 pictures that were also presented as targets (TPs). Four pictures showed target animals in a different illustration as the targets, i.e. as black line drawings depicting the same animals but drawn different in contour as well as in shade of color (Diff-Illu-TP). Two of the animal primes in different illustration had the same left-right orientation as the target pictures while the orientation of the other two Diff-Illu-TPs was left-right reversed.

\footnotetext{
${ }^{1}$ We thank Bruno Rossion, Gilles Pourtois, and Michael J. Tarr for making the pictures available (see homepage of Michael J. Tarr, Brown University, http://www.tarrlab.org/).
} 
Four pictures showed different animals (camel, bear, bird, and ant) that were never presented as targets (Same-Cat-NP), and four pictures showed the objects bed, house, pliers, and key (Diff-CatNP). The 64 possible prime-target combinations were presented 10 times each. After each 80 trials, participants were allowed a short, self-paced break. The pictures were drawn in a white rectangle extending $2 \mathrm{~cm}$ high by $3 \mathrm{~cm}$ wide. Masks were random dot patterns extending $7.5 \times 7.5 \mathrm{~cm}$. They were constructed such that always $4 \times 4$ pixels were chosen randomly to be white or black. To increase masking, we presented always five different random dot patterns with a total duration of $72 \mathrm{~ms}(5 \times 14.3 \mathrm{~ms})$ as preand postmask. Additionally, the prime picture as well as the target picture was also presented with a random dot pattern frame.

The sequence of the events in a trial is shown in Figure 1. On each trial, a fixation cross was presented for $200 \mathrm{~ms}$. Then the premask was presented for $72 \mathrm{~ms}$ followed by the prime presented for $28 \mathrm{~ms}$ (two refresh cycles) and the postmask presented for 72 $\mathrm{ms}$. At last, the target was presented directly after the postmasks for $250 \mathrm{~ms}$.

\section{Design and Procedure}

Participants were instructed to categorize the depicted animals as being smaller or larger than a box (measuring $51 \times 50 \times 24 \mathrm{~cm}$ ) that was placed next to the monitor. Half of the participants pressed a left key with the left index finger to indicate "smaller" and a right key with the right index finger to indicate "larger" as fast and as accurately as possible. For the other half, the mapping was reversed. Errors were indicated by the German word for wrong ("Falsch!") presented in red in the lower part of the monitor Response times (RTs) were recorded from the onset of the target until the onset of the response.

At the end of the experiment, we tested prime visibility. Participants were fully informed about the precise structure of the prime stimuli. All prime pictures were shown on a separate sheet of paper which remained besides the keyboard during the discrimination task. Then, 80 trials identical to the experimental trials were presented and participants were asked to discriminate whether the prime picture was smaller or larger than the reference object. For the discrimination task, participants were instructed to take their time and to try to be as accurate as possible. In order to avoid that unconsciousness congruency effects influence the free response choice (adapted after Vorberg, Mattler, Heinecke, Schmidt, \& Schwarzbach, 2003; see also Kiesel, Wagener et al., 2006; Schlaghecken \& Eimer, 2004), there was an interval of $800 \mathrm{~ms}$ after target offset, in which no response was possible.

\section{Results}

\section{Congruency Effect}

Trials with RTs deviating more than 2.5 SDs from the mean RT of each participant and each condition were excluded.

Mean RTs for correct responses were submitted to an analysis of variance (ANOVA) with the within-subject factors Prime Type (TP, Diff-Illu-TP, Same-Cat-NP and Diff-Cat-NP) and Congruency (congruent and incongruent). Averaged data across all par-

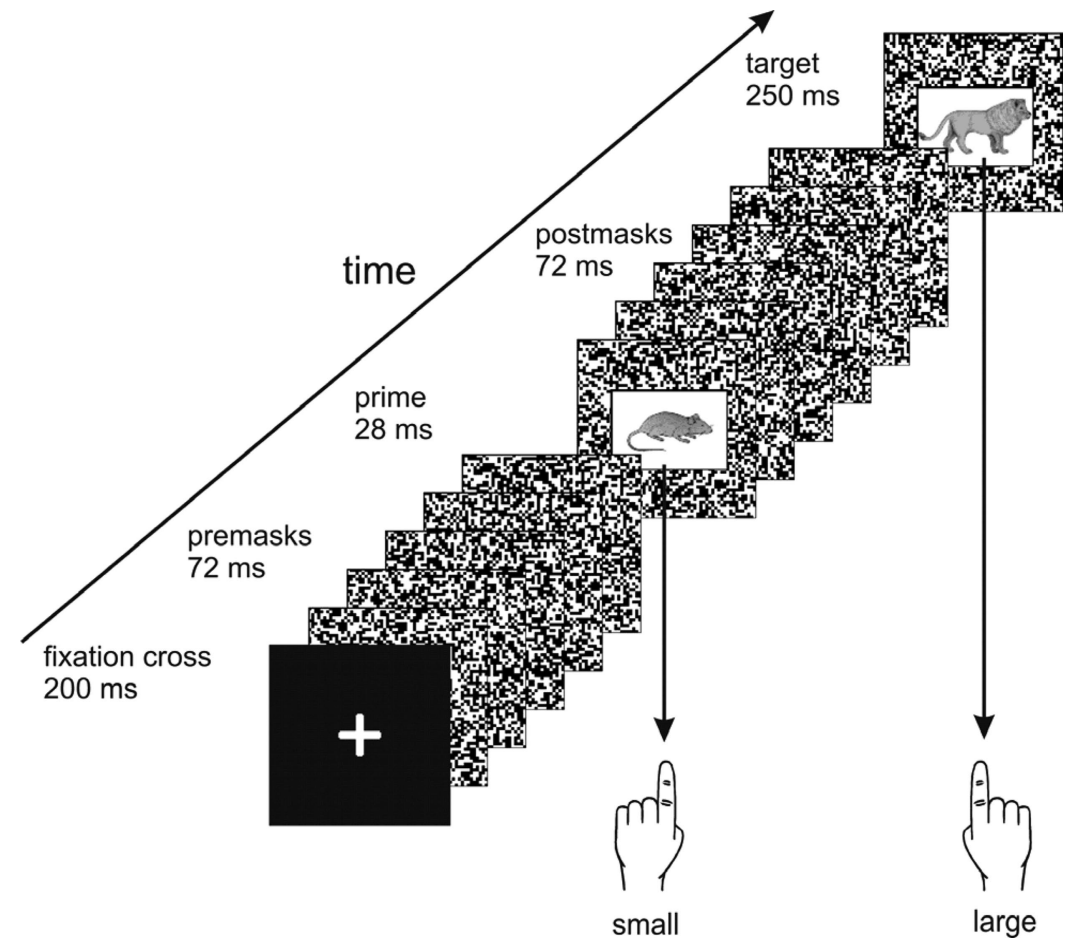

Figure 1. Sequence of the events in Experiment 1. The Figure shows an incongruent trial because the prime picture (the mouse) would afford the response indicating "small" while the target picture (the lion) affords the alternative response indicating "large." 
ticipants are presented in Figure 2. The factor Congruency was significant, $F(1,23)=30.1, p<.001, \eta^{2}=.57, M S E=6,501.4$, as well as the interaction Prime Type $\times$ Congruency $F(3,69)=$ 13.0, $p<.001, \eta^{2}=.37, M S E=1,915.6$. The main effect for Prime Type did not approach significance, $p>.13$. Single comparisons (see Figure 2) revealed that only TPs $t(23)=6.56, p<$ .001 and Diff-Illu-TPs $t(23)=3.15, p<.01$ elicited a congruency effect. Participants responded faster to congruent (395 ms) rather than incongruent (425 ms) TPs and faster to congruent (402 ms) rather than incongruent (413 ms) Diff-Illu-TPs. However, a separate and more detailed analysis of Diff-Illu-TPs revealed that only the two prime pictures with the same left-right orientation as the target pictures did elicit a congruency effect $t(23)=3.94, p<$ .001 , whereas the other two prime pictures with a reversed leftright orientation as the target pictures did not, $p>.27$. Also the other two prime types did not reach significance. There was no reliable congruency effect for Same-Cat-NPs, $p>.18$ as well as for Diff-Cat-NPs, $p>.76$.

The overall mean error rate was $6.1 \%$. The same ANOVA on error rates revealed significant effects for the factor Prime Type, $F(3,69)=2.8, p<.05, \eta^{2}=.11, M S E=.002$, the factor Congruency $F(1,23)=9.7, p<.01, \eta^{2}=.30, M S E=.021$, and the interaction Prime Type $\times$ Congruency, $F(3,69)=7.9, p<$ $.001, \eta^{2}=.26, M S E=.008$. Single comparisons show that again only TPs, $t(23)=4.11, p<.001$ with $9.6 \%$ errors in incongruent compared to $4.5 \%$ errors in congruent trials and Diff-Illu-TPs, $t(23)=2.38, p<.05$ with $7.3 \%$ errors in incongruent compared to $4.4 \%$ errors in congruent trials yielded a congruency effect. Again, a separate and more detailed analysis of Diff-Illu-TPs revealed that only the two prime pictures with the same left-right

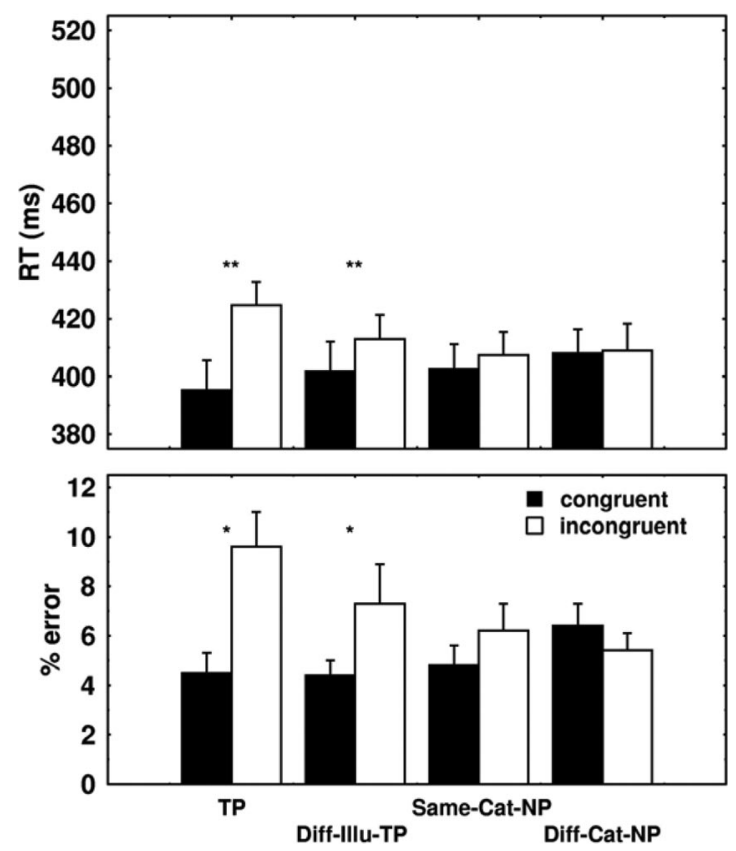

Figure 2. Mean response times (RTs; upper panel) and error rates (lower panel) depending on Prime Type and Congruency in Experiment 1. Lines represent standard errors. Asterisks indicate the significance level: * $p<.05$. ${ }^{* * *} p<.01$. orientation as the target pictures elicited a congruency effect $t(23)=2.74, p<.05$, whereas the other two prime pictures with a reversed left-right orientation as the target pictures did not elicit a significant congruency effect, $p>.28$. Also, the congruency effect for the other two prime types did not reach significance. There was no reliable congruency effect for Same-Cat-NPs, $p>$ .11 as well as for Diff-Cat-NPs, $p>.21$.

\section{Prime Visibility}

To assess prime visibility, we computed the signal detection measure $d^{\prime}$. Prime pictures requiring a small response were treated as signal, whereas prime pictures requiring a large response were considered as noise. Hits and false alarms proportion of zero or one were corrected according to the log-linear rule (Goodman, 1970; cited according to Hautus, 1995) if participants had $0 \%$ hits or $100 \%$ false alarms. Overall discrimination for prime pictures was $d^{\prime}=.42$ (the mean hit rate was $55.5 \%$, false alarm rate was $39.9 \%$ ) and deviated from zero, $t(23)=4.62, p<.001$.

A one-way ANOVA with the factor Prime Type (TP, Diff-IlluTP, Same-Cat-NP and Diff-Cat-NP) revealed that Prime Type had a significant effect on prime visibility, $F(3,69)=5.37, p<.01$, $\eta^{2}=.19, M S E=1.4$. Separately for the different prime types, discrimination performance was for TPs $d^{\prime}=.79, t(23)=5.83$, $p<.001$, for Diff-Illu-TPs $d^{\prime}=.41, t(23)=3.35, p<.01$, for Same-Cat-NPs $d^{\prime}=.32, t(23)=2.29, p<.05$, and for Diff-CatNPs $d^{\prime}=.25, t(23)=1.90, p>.07$.

To test whether the congruency effect is related to the prime visibility, a regression analysis as proposed by Draine and Greenwald (1998, see also Greenwald, Klinger, \& Schuh, 1995; Greenwald et al., 1996) was computed. A priming index was calculated for each participant and each prime type, with index $=100 \times(\mathrm{RT}$ incongruent - RT congruent)/RT congruent. Individual priming indices for the four prime types were regressed onto the four individual $d^{\prime}$ values for the four prime types. The linear regression analysis revealed no significant correlation between $d^{\prime}$ and the priming index for TPs with $r=.110, F(1,23)=.27, p>.60$, for Same-Cat-NPs with $r=.208, F(1,23)=.99, p>.33$ and Diff-Cat-NPs with $r=.189, F(1,23)=.82, p>.37$. For Diff-Illu-TPs the linear regression showed a significant correlation between $d^{\prime}$ and the priming index with $r=.426 F(1,23)=4.88$, $p<.05$. The intercept of the regression was larger than zero for TPs (intercept $=7.21, t(23)=3.70, p<.01$ ), indicating that the significant congruency effect for TPs is associated with a $d^{\prime}$-value of zero. In contrast, there was no significant intercept for DiffIllu-TPs (intercept $=1.78, t(23)=1.69, p>.10$ ), for Same-CatNPs (intercept $=1.32, t(23)=1.01, p>.33$ ) and for Diff-Cat-NPs (intercept $=-.09, t(23)=-.12, p>.90)$.

Thus, the observed congruency effect for primes picturing animals seen as targets (TPs) is independent on individual prime visibility. In contrast, the positive correlation of prime visibility and congruency effect for prime pictures presented in a different illustration as the targets (Diff-Illu-TPs) as well as the fact that the intercept does not differ significantly from zero for these primes indicates that these primes do not reveal a reliable congruency effect related to null visibility. 


\section{Discussion}

In Experiment 1, reliably congruency effects were observed for prime pictures that were also used as targets (TPs). Prime pictures that depicted the same animals as the target pictures but were drawn in a different illustration (Diff-Illu-TPs) only elicited priming when they were drawn with the same left-right orientation as the target pictures. Diff-Illu-TPs in the opposite left-right orientation were ineffective as well as prime pictures of animals never presented as targets (Same-Cat-NP) and prime pictures of objects (Diff-Cat-NP). Thus, the results in Experiment 1 replicate the finding that priming is restricted to target primes or primes that are perceptually very similar to the targets when the target set is small (e.g., Abrams \& Greenwald, 2000, Exp. 3; Damian, 2001; Kiesel, Kunde, Pohl, \& Hoffmann, 2006). Moreover, Experiment 1 extends this finding in two aspects: First, there was no transfer to novel primes although primes and targets derived from a semantically closely related category like animals. Second, a change in the perceptual appearance-depicting animals slightly differently and in black line drawings instead of grey scale-shaded images and presenting them in opposite left-right orientation-already sufficed to eliminate priming. So, Experiment 1 demonstrates early selection of unconscious information processing.

Regarding prime visibility, the detection rate differed significantly from zero. Thus, the masking procedure in Experiment 1 did not completely prevent prime visibility. However, we found no significant correlation between prime impact on the target response and prime visibility for TPs. Additionally, the significant positive intercepts indicate that priming was present even when prime visibility was at zero. Interestingly, discrimination performance for primes picturing targets (TPs) was higher than discrimination performance for the other prime types and these primes elicited the strongest congruency effects. First, one might suspect that the pictures used as TPs were easier to discriminate leading to higher prime visibility as well as higher (and significant) difference between congruent and incongruent trials. To test whether target pictures were more discriminable per se, we reran the discrimination task with a new group of 12 participants. Now, Prime Type had no significant effect on prime visibility $d^{\prime}, F(3$, $33)<1$ (TPs $d^{\prime}=.67, t(11)=2.63, p<.05$, Diff-Illu-TPs $d^{\prime}=$ $.54, t(11)=2.56, p<.05$., Same-Cat-NPs $d^{\prime}=.44, t(11)=1.67$, $p>.12$, and Diff-Cat-NPs $d^{\prime}=.27, t(11)=1.19, p>.26$.).

To account for this finding, we conjecture that participants discriminated the target primes more easily, because their former intentions of the main experiment still prevailed or because participants had built up better perceptual representations of the target pictures through practice in the main experiment. Currently, we cannot decide between these two alternatives. However, a blockwise analysis of the congruency effects in Experiment 1 might speak to this issue. ${ }^{2}$ If the larger congruency effect/higher visibility for target primes is due to the fact that they are easier to discriminate because they are shown many times as a target, then one might expect a gradual increase of the priming effect as a function of blocks. Alternatively, when the larger congruency effect/higher visibility is due to increased attention for those stimuli that were relevant for the intended action in the main experiment, congruency effects should remain similar over blocks, since intentions can be formed almost immediately. An ANOVA with the withinsubject factors Block (one to eight) and Congruency (congruent and incongruent) only for TPs revealed no increase of the congruency effect; the interaction was neither significant for RTs $F(7$, $161)=1.3, p>.26, \eta^{2}=.05, M S E=1,748.7$, nor for errors $F(7$, $161)<1$. This result might be taken as a cautious hint that target primes were better visible because the former intentions prevailed. Yet a blockwise analysis might not be sufficient to rule out practice effects because with only four targets few trials might be sufficient to maximize performance.

This theoretically interesting explanation is based on the claim that the function of attention is to select stimuli that are relevant for an intended action (Allport, 1987). Therefore, perception for expected stimuli is fostered while even clearly visible but unexpected stimuli may be overseen (cf. Mack \& Rock, 1998). Such a mechanism might account for the different visibility scores obtained in Experiment 1: In the main categorization task, only the four target pictures were relevant for participants' response and discriminating these pictures was well trained. In the discrimination task, participants had to discriminate these target pictures as well as novel pictures. This result fits well to recent studies showing that top down modulated attention influences not just the effects of priming but also the effects of masking (Boyer \& Ro, 2007; Enns $\&$ Oriet, 2007). Yet, such a conclusion is still speculative and because it is not the main point of our study, we defer to further research to examine this conjecture.

\section{Experiment 2}

The conditions in Experiment 1 induced early selection of masked stimuli. Experiment 2 explored conditions that might prompt a late selection. Experiencing a larger target set should change representations of and expectations about possible target stimuli. When there are many different target stimuli, preparation for single stimuli is no longer possible or at least very demanding. Consequently, the conceptual or semantic meaning of the stimuli is likely to be considered to perform the categorization task. Such a task setting would require late selection mechanisms because stimuli's conceptual or semantic meaning are needed for task performance.

In Experiment 2, we enlarged the target set from 4 to 40 target pictures of animals while using the same primes as in Experiment 1. Again, the instructed task was a size classification. As in Experiment 1, using the four different types of primes allowed us to assess systematically whether masked primes were selected early or late. If priming were again restricted to target primes and primes that are perceptually very similar to the targets, masked primes would be subject to early selection despite of the enlarged target set. In contrast, if priming spread to novel primes, masked primes were selected late. For this case, two further alternatives might be distinguished. First, all prime stimuli might induce congruency effects because they can be easily classified as small or large. Thus, each masked prime was semantically classified and no selection at all happened. Alternatively, masked priming may spread to novel primes, but be restricted to animal primes (i.e. Diff-Illu-TPs and Same-Cat-NPs) because participants are taking other animals as potential targets into account, but not objects from other semantic categories.

\footnotetext{
${ }^{2}$ We thank an anonymous reviewer for suggesting this analysis.
} 
Priming confined to all primes picturing animals would reveal that not each appropriate stimulus is processed according to the task instructions but that the masked stimuli are selected based on their membership to the category "animal."

\section{Method}

\section{Participants}

Twenty-four students (eight males) of the University of Würzburg with an average age of 23.8 years participated in Experiment 2 in fulfilment of course requirements. All reported having normal or corrected-to-normal vision, and were not familiar with the purpose of the experiment. An experimental session lasted approximately $45 \mathrm{~min}$.

\section{Apparatus and Stimuli}

Apparatus and stimuli were identical to Experiment 1 except that the target set was enlarged to 40 animal pictures. Twenty pictures of small animals (mouse, chicken, lobster, sea horse, fly, cat, skunk, snail, bee, squirrel, duck, fish, frog, spider, grasshopper, beetle, caterpillar, turtle, snake, and butterfly) and 20 pictures of large animals (lion, zebra, giraffe, gorilla, penguin, tiger, pig, monkey, donkey, goat, dog, leopard, kangaroo, alligator, cow, rhinoceros, horse, deer, seal, and sheep) served as targets. As primes, the same 16 stimuli were used as in Experiment 1. The pictures used as primes and targets appeared in a white rectangle $2 \mathrm{~cm}$ high by $3 \mathrm{~cm}$ wide (seven target pictures were $3 \mathrm{~cm}$ high by $2 \mathrm{~cm}$ wide). Each prime-target-pair was presented once in the experiment resulting in 640 trials (16 primes $\times 40$ targets).

\section{Design and Procedure}

Design and procedure were identical to Experiment 1.

\section{Results}

\section{Congruency Effect}

Trials with RTs deviating more than 2.5 SDs from the mean RT of each participant and each condition were excluded. Mean RTs and PEs were computed for each participant for each combination of the factors Prime Type (TP, Diff-Illu-TP, Same-Cat-NP and Diff-Cat-NP) and Congruency (congruent and incongruent). Averaged data across all participants are presented in Figure 3.

Mean RTs for correct responses were submitted to an ANOVA with the within-subject factors Prime Type and Congruency. The factor Congruency was significant, $F(1,23)=56.4$, $p<.001, \eta^{2}=.71, M S E=17,489.5$, as well as the interaction Prime Type $\times$ Congruency, $F(3,69)=10.3, p<.001, \eta^{2}=$ $.31, M S E=1,925.8$. Single comparisons revealed congruency effects for all primes picturing animals (see Figure 3): Participants responded faster for congruent ( $457 \mathrm{~ms}$ ) than incongruent $(483 \mathrm{~ms})$ TPs, $t(23)=6.11, p<.001$, for congruent $(463 \mathrm{~ms})$ than incongruent (485 ms) Diff-Illu-TPs, $t(23)=4.23, p<$ .001 , and for congruent $(460 \mathrm{~ms})$ than incongruent $(488 \mathrm{~ms})$ Same-Cat-NPs, $t(23)=6.57, p<.001$. There was no congru-
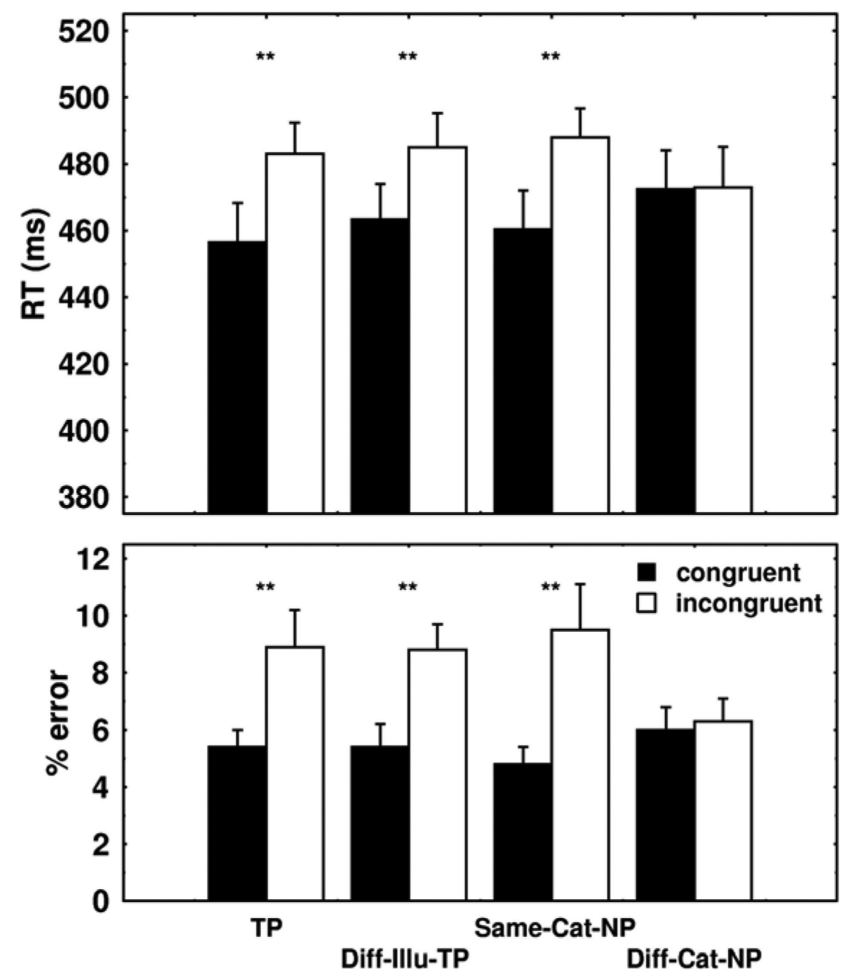

Figure 3. Mean response times (RTs; upper panel) and error rates (lower panel) depending on Prime Type and Congruency in Experiment 2. Lines represent standard errors. Asterisks indicate the significance level: ${ }^{*} p<$ .05. ${ }^{* *} p<.01$.

ency effect for Diff-Cat-NPs, $p>$.87. A separate and more detailed analysis of Diff-Illu-TPs revealed that all Diff-Illu-TPs elicited a congruency effect irrespective of their orientationthe two prime pictures with the same left-right orientation as the target pictures, $t(23)=3.41, p<.01$, as well as the other two prime pictures with a reversed left-right orientation as the target pictures, $t(23)=3.67, p<.01$, revealed congruency effects.

The overall mean error rate was $6.9 \%$. The pattern of the error rates mirrors the RT data. The same ANOVA on errors revealed significant effects for the factor Congruency $F(1,23)=15.0, p<$ $.001, \eta^{2}=.40, M S E=.044$, and the interaction Prime Type $\times$ Congruency, $F(3,69)=3.9, p<.05, \eta^{2}=.14, M S E=.012$. Single comparisons of the error rates revealed congruency effects for all primes picturing animals (see Figure 3): Participants made more errors with incongruent $(8.9 \%)$ compared to congruent $(5.3 \%)$ TPs, $t(23)=3.09, p<.01$, with incongruent $(8.8 \%)$ compared to congruent $(5.4 \%)$ Diff-Illu-TPs, $t(23)=4.07$, $p<.001$, and with incongruent $(9.5 \%)$ compared to congruent $(4.8 \%)$ Same-Cat-NPs, $t(23)=3.09, p<.01$. There was no congruency effect for Diff-Cat-NPs, $p>$.65. A separate and more detailed analysis of Diff-Illu-TPs revealed that all Diff-Illu-TPs elicited a congruency effect irrespective of their orientation - the two prime pictures with the same left-right orientation as the target pictures $t(23)=3.59, p<.01$, as well as the other two prime pictures with a reversed left-right orientation as the target pictures $t(23)=2.97, p<.01$. 


\section{Prime Visibility}

Overall discrimination for prime pictures was $d^{\prime}=.40$ (the mean hit rate was $56.8 \%$, false alarm rate $41.6 \%$ ) and deviated from zero, $t(23)=3.84, p<.001$. Separately for the different prime types, the discrimination performance was: for TPs $d^{\prime}=.64$, $t(23)=5.40, p<.001$, for Diff-Illu-TPs $d^{\prime}=.14, t(23)=1.02$, $p>.31$, for Same-Cat-NPs $d^{\prime}=.66, t(23)=3.60, p<.001$, and for Diff-Cat-NPs $d^{\prime}=.27, t(23)=1.82, p>.82$. Mean $d^{\prime}$-values for each participant were subjected to a one-way ANOVA with the factor Prime Type (TP, Diff-Illu-TP, Same-Cat-NP and Diff-CatNP). It revealed that Prime Type had a significant effect on prime visibility $d^{\prime}, F(3,69)=5.13, p<.01, \eta^{2}=.18, M S E=1.6$.

To test whether the priming effect is related to prime visibility, a priming index for the RT congruency effect was computed for each participant and each prime type as in Experiment 1. A subsequently performed linear regression analysis revealed no significant correlation between the four individual $d^{\prime}$ values and the priming index for TPs with $r=.067, F(1,23)=.10, p>.75$, for Diff-Illu-TPs with $r=$ $.245, F(1,23)=1.41, p>.24$, for Same-Cat-NPs with $r=.356, F(1$, $23)=3.18, p>.08$, and Diff-Cat-NPs with $r=.050, F(1,23)=.05$, $p>$.81. The intercept of the regression was larger than zero for TPs (intercept $=5.89, t(23)=3.70, p<.001$ ), for Diff-Illu-TPs (intercept $=4.61, t(23)=4.0, p<.001$ ), and for Same-Cat-NPs (intercept $=5.19, t(23)=4.21, p<.001)$, but not for Diff-Cat-NPs (intercept $=.04, t(23)=.06, p>.95)$. Thus, significant priming effects are associated with $d^{\prime}$-values of zero, and the observed priming effects for all primes picturing animals do not significantly depend on individual prime visibility.

\section{Discussion}

Participants responded significantly slower in incongruent compared to congruent trials when the prime stimuli were animals irrespective of whether the animal pictures had been presented as targets or not. In contrast, primes picturing objects did not induce congruency effects. The designs of Experiment 1 and 2 differentiated only regarding the number of targets. This - on a first sight smallish-manipulation led to important differences in the obtained pattern of results.

Yet, before addressing these differences in the pattern of results, a closer look at the differences in the experimental designs is necessary. In Experiment 2, participants had to respond to many (40) targets picturing animals, whereas in Experiment 1 only 4 targets were presented. One might conjecture that the pictures in this large target set of animal stimuli in Experiment 2 entailed more feature overlap with the novel primes than did the small target set of Experiment 1. ${ }^{3}$ This could be crucial because there are several findings demonstrating that similarity between the perceptual features of prime and target can facilitate responses even independently on prime-target response congruence. For example, in experiments in which parts of the primes shared perceptual features of targets associated with the other response, paradoxical congruency effects were observed (Abrams \& Greenwald, 2000; Greenwald et al., 2003). These findings show that the impact of similar perceptual features between primes and target can even be stronger than the influence of an instructed semantic categorization. However, as stated in the introduction, priming due to elemental perceptual features would be a clear demonstration of early selection and disqualifies a late selection interpretation.
To rule out that different amounts of feature overlap caused the different result patterns of Experiment 1 and 2, we ran another analysis of Experiment 2 restricted to trials with the same four targets as in Experiment 1. The prime sets were identical in Experiment 1 and 2, and thus the prime-target feature overlap with this subsample of targets was identical as well. Participants responded faster for congruent (446 ms) than incongruent (469 ms) TPs, $t(23)=2.42, p<.05$, and for congruent $(448 \mathrm{~ms})$ than incongruent (479 ms) Same-Cat-NPs, $t(23)=3.08, p<.01$. The difference between congruent (451 ms) compared to incongruent (471) Diff-Illu-TPs almost reached significance, $t(23)=2.07, p<$ .051 . There was no congruency effect for Diff-Cat-NPs, $p>.80$. A separate and more detailed analysis of Diff-Illu-TPs revealed that the two prime pictures with the same left-right orientation as the target pictures did not reveal a significant effect $p>.24$ (455 $\mathrm{ms}$ for congruent compared to $470 \mathrm{~ms}$ for incongruent trials), but the other two prime pictures with a reversed left-right orientation as the target pictures produced a significant congruency effect $t(23)=2.23, p<.05$ (447 ms for congruent compared to $480 \mathrm{~ms}$ for incongruent trials).

Thus, we observed a similar pattern of results when we restricted the analysis to trials with the same target pictures as in Experiment 1. In these trials, there was no difference in the amount of feature overlap between primes and targets in Experiment 1 and 2. Nonetheless, not only TPs revealed reliable congruency effects, but also novel primes picturing animals (Same-Cat-NPs) and Diff-Illu-TPs with a reversed left-right orientation induced congruency effects.

Enlarging the target set from four targets in Experiment 1 to forty targets in Experiment 2 leads to three main differences in the pattern of results:

Firstly, participants responded in average $65 \mathrm{~ms}$ slower and made $.8 \%$ more errors in Experiment 2 than in Experiment 1. Obviously, responding was more difficult with the large compared to the small target set. This considerable difference in reaction times indicates that our manipulation of processing depth for the targets worked. Presumably, a deeper processing of the targets requires more processing time and results in longer response times.

Secondly, in Experiment 2 we observed congruency effects for all primes depicting animals irrespective of whether they were presented as targets or not, whereas in Experiment 1 congruency effects were restricted to target primes and primes in a different illustration (Diff-Illu-TPs) drawn with the same left-right orientation as the target pictures. A between-experiment comparison of the congruency effects of primes picturing greyscale shaded images of animals that were not presented as targets (Same-Cat-NPs) revealed that for RTs the size of the congruency effect in Experiment $2(28 \mathrm{~ms})$ differed significantly from the size of the congruency effect in Experiment 1 ( $5 \mathrm{~ms}), t(23)=4.10, p<.001$; for errors, the difference between the size of congruency effect in Experiment $2(4.8 \%)$ and in Experiment $1(1.4 \%)$ was marginally significant $t(23)=1.93, p<.06$. Similarly, the same comparison for Diff-Illu-TPs in the opposite left-right orientation revealed that for RTs the size of the congruency effect in Experiment 2 (21 ms) differed significantly from the size of the congruency effect in Experiment $1(6 \mathrm{~ms}), t(23)=2.32, p<.05$; for errors the

\footnotetext{
${ }^{3}$ We thank Tony Greenwald for pointing out this important aspect.
} 
difference between the size of congruency effect in Experiment 2 $(3.6 \%)$ and in Experiment $1(1.7 \%)$ was not significant, $p>.33$.

Thus, masked stimuli were no longer selected based on their sensory features but based on their semantic or conceptual meaning. This finding is relevant because evidence for a transfer of priming to novel primes is still rare. Whereas transfer of novel primes has been observed several times with word stimuli (Kiesel, Kunde, \& Hoffmann, 2006; Klauer et al., 2006; Naccache et al., 2005) as well as with number stimuli (Kunde et al., 2003; Naccache \& Dehaene, 2001), the present Experiment is a rare replication of a frequently cited study that used picture primes (Dell'Acqua \& Grainger, 1999; see Van den Bussche, Notebaert, $\&$ Reynvoet, 2009, for the only other replication we are aware of).

More important, Experiment 2 extends these findings regarding two important aspects. On the one hand, novel animal pictures (Diff-Illu-TPs and Same-Cat-NP) influenced target responding according to the size classification task. Therefore, the conceptual meaning of the pictured animals as small or large must have been processed. Considering the size of the animals pictured as primes in real life requires much more than relying on a mere perceptual feature. So, primes were subject to late selection here. On the other hand, previous studies reported congruency effects for all novel primes suggesting that any appropriate stimulus was processed. Contrarily, in Experiment 2 novel primes picturing objects (DiffCat-NPs) failed to evoke congruency effects, although these primes could have been easily classified as smaller or larger than the reference object. Thus, when only animals were presented as targets, object primes had no impact through their semantic congruence and despite that task instruction could be applied to them. That is, not each appropriate masked stimulus was processed but only stimuli that belong to the same semantic category as the experienced targets. The observation that novel primes picturing animals induced congruency effects whereas novel primes picturing objects were ineffective is a clear demonstration of the impressive ability of the cognitive system to select even masked stimuli at a late stage.

Like in Experiment 1, Prime Type had a significant influence on discrimination performance, indicating that visibility for Same-CatNPs and TPs was higher than for the other prime types. The almost equal detection rate for Same-Cat-NPs and TPs might be due to participants prevailing intentions from the main categorization task. To rule out that the Same-Cat-NPs and TPs were better visible in general when applying this target set, a new group of 12 participants who had not taken part in the main experiment performed the same discrimination task. Their data did not reveal a significant impact of Prime Type on prime visibility $d^{\prime}, F(3,33)=1.12, p>.35, \eta^{2}=.09$, $M S E=0,517$, (TPs $d^{\prime}=.60, t(11)=2.76, p<.05$, Diff-Illu-TPs $d^{\prime}=.35, t(11)=1.70, p>.11$., Same-Cat-NPs $d^{\prime}=.09, t(11)=.39$, $p>.70$, and Diff-Cat-NPs $\left.d^{\prime}=.34, t(11)=2.00, p>.07.\right)$, supporting our conjecture that prevailing intentions led to higher visibility for the expected primes. Like for Experiment 1, a blockwise analysis of the congruency effects revealed no impact of training on the size of the congruency effect. The interaction of block $x$ congruency was neither significant for RTs $F(7,161)<1$, nor for errors $F(7$, $161)<1$ for TPs, and it was neither significant for RTs $F(7,161)=$ $1.6, p>.12, \eta^{2}=.07, \operatorname{MSE}=3,711.6$, nor for errors $F(7,161)=$ $1.13, p>.34, \eta^{2}=.05, M S E=.008$, for NPs. Thus, this result suggests that training of specific stimuli in the main experiments leads to increased visibility scores in the discrimination task.

\section{Experiment 3}

In Experiment 2, the large target set consisting just of animal pictures led to late selection of masked stimuli. Next, we wanted to elaborate whether such a late selection mechanism may be influenced by a second semantic category that corresponds to the semantic category instructed for response selection. Therefore, we introduced an additional feature of the experienced targets that was not relevant for the instructed task but covaried with the required response. The target set in Experiment 3 was constructed such that in addition to the feature for the instructed categorization, target stimuli differed in an apparent second feature. The task required a small/large-classification and the target set consisted of 40 pictures. All target pictures requiring a "small" response were objects and all pictures requiring a "large" response were animals. Thus, the semantic affiliation "animal" or "object" correlated with the likewise required response "small" or "large." Whereas animate/ inanimate distinctions are well established in long-term memory, possibly even at a neuronal level (Farah, 1994), the distinction of objects according to an arbitrary size is certainly not. We therefore conjecture that responding would be easier when participants implicitly or explicitly relied on the response-contingent but not instructed animate/inanimate distinction instead of the actually instructed small/large distinction.

As primes, 12 pictures were used: 4 pictures that were also presented as targets (TP), 4 pictures that were never presented as targets but were also large animals or small objects (Same-Cov$\mathrm{NP}$ ), and 4 pictures with reversed co-variation, that is, 2 pictures of small animals (Small-Animal-NP) and 2 pictures of the large objects (Large-Object-NP). Because we used a large target set, we expected congruency effects for primes presented as targets (TPs) as well as for primes never seen as targets but being also large animals or small objects (Same-Cov-NPs). The crucial primes are the ones with inversed covariation, i.e. large objects and small animals. The decisive question is whether these primes are selected according to the instructed or according to the covarying feature. As long as participants categorize the prime stimuli according to their size, primes picturing small animals for example should facilitate the response to targets picturing small objects. However, when the covarying feature-the categorization between animal and object-is incorporated in action control then primes picturing small animals should be selected for the large response and facilitate the response to targets picturing large animals. This outcome would show up as a reversed congruency effect regarding the size categorization task.

\section{Method}

\section{Participants}

Twenty-four students (seven males) of the University of Würzburg with an average age of 21.5 years participated in Experiment 3 in fulfilment of course requirements. All reported having normal or corrected-to-normal vision, and were not familiar with the purpose of the experiment. An experimental session lasted approximately $35 \mathrm{~min}$.

\section{Apparatus and Stimuli}

Apparatus and stimuli were similar to Experiment 1 except for the following changes: Targets were 20 pictures of small objects 
(book, spool of thread, thimble, lightbulb, nail, button, shoe, clothespin, fork, watch, football, pipe, cup, lock, trumpet, hat, telephone, scissors, hammer and gun) and 20 pictures of large animals (lion, zebra, giraffe, gorilla, penguin, tiger, pig, monkey, donkey, goat, dog, leopard, kangaroo, alligator, cow, rhinoceros, horse, deer, seal and sheep). Twelve pictures were used as primes: 4 pictures (zebra, lion, fork and button) that were also presented as targets (TP), 4 pictures (camel, bear, pliers and key) that were never presented as targets but were also large animals or small objects (Same-Cov-NP), and 4 pictures with reversed covariation, that is 2 pictures (mouse and snail) of small animals (SmallAnimal-NP) and 2 pictures (bed and house) of large objects (Large-Object-NP).

\section{Design and Procedure}

The instruction and procedure was similar to Experiment 1 . When instructing the task, we took care that the covariation between size and category was never mentioned. The experiment consisted of 480 trials with each prime-target pair presented once.

In a postexperimental prime discrimination task, 84 trials were presented.

\section{Results}

\section{Congruency Effect}

Trials with RTs deviating more than 2.5 SDs from the mean RT of each participant and each condition were excluded. Mean RTs and PEs were computed for each participant and each combination of the factors Prime Type (TP, Same-Cov-NP, Small-Animal-NP and Large-Object-NP) and Congruency (congruent and incongruent). Averaged data across all participants are presented in Figure 4.

Mean RTs for correct responses were submitted to an ANOVA with the within-subject factors Prime Type and Congruency. The interaction Prime Type $\times$ Congruency was significant, $F(3,69)=$ 19.5, $p<.001, \eta^{2}=.46, M S E=26,536.4$. The main effect Congruence was significant $F(1,23)=5.0, p<.05, \eta^{2}=.18$, $M S E=1,904.9$, whereas the factor Prime Type failed to reach significance, $p>06$. Single comparisons revealed congruency effects for TPs, $t(23)=7.36, p<.001$, and Same-Cov-NPs, $t(23)=4.51, p<.001$. Participants responded faster for congruent (428 ms) than incongruent (456 ms) TPs, as well as for congruent (425 ms) than incongruent (445 ms) Same-Cov-NPs. Contrarily, the congruency effect for Small-Animal-NPs was reversed with respect to the instructed small/large-classification, $t(23)=-5.86$, $p<.001$. Here, participants responded faster for incongruent (420 $\mathrm{ms})$ compared to congruent (453 ms) Small-Animal-NPs. There was no congruency effect for Large-Object-NPs $(p>.25)$.

The overall mean error rate was $6.5 \%$. The same ANOVA on error rates almost mirrored the RT data. The interaction Prime Type x Congruence was significant, $F(3,69)=6.3, p<$ $.001, \eta^{2}=.12, M S E=.037$, as well as the factor Congruence $F(1$, $23)=10.07, p<.01$. The factor Prime Type was not significant $p>$.10. Single comparisons show that only TPs, $t(23)=4.32, p<$ .001 , and Same-Cov-NPs, $t(23)=2.76, p<.01$, elicited significant congruency effects, indicating that participants made more errors for incongruent $(10.0 \%)$ compared to congruent $(4.8 \%)$ TPs and for incongruent $(8.1 \%)$ compared to congruent $(5.2 \%)$ Same-
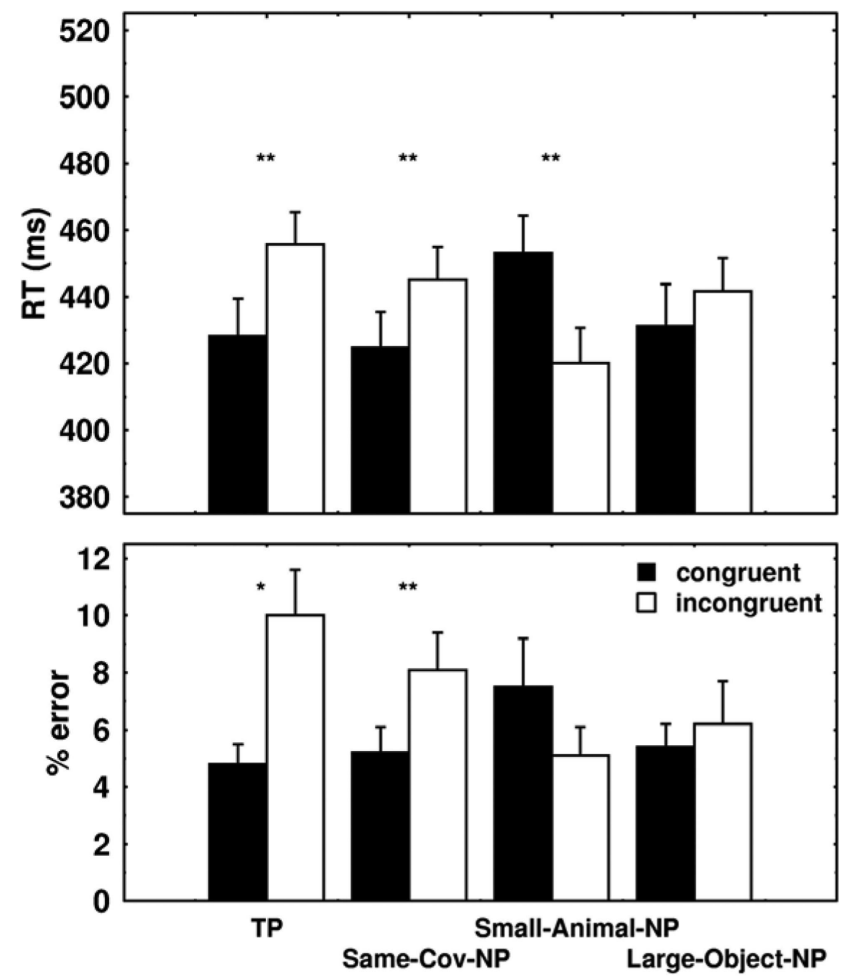

Figure 4. Mean response times (RTs; upper panel) and error rates (lower panel) depending on Prime Type and Congruency in Experiment 3. Lines represent standard errors. Asterisks indicate the significance level: ${ }^{*} p<.05$ *** $p<.01$

Cov-NPs. The other prime types did not induce significant congruency effects $(p s>.50$ for Large-Object-NPs und $p s>.11$ for Small-Animal-NPs with a negative t-value of $t(23)=-1.65$, because participants made $5.1 \%$ errors with incongruent compared to $7.5 \%$ errors with congruent Small-Animal-NPs).

\section{Prime Visibility}

Overall discrimination performance was $d^{\prime}=.69$ (the mean hit rate was $62.0 \%$, false alarm rate $36.0 \%$ ) and deviated from zero, $t(23)=$ $8.10, p<.001$. Separately for the different prime types, $d^{\prime}$ was for TPs $d^{\prime}=1.26, t(23)=10.05, p<.001$, for Same-Cov-NPs $d^{\prime}=.54$, $t(23)=4.27, p<.001$ and for primes with a different co-variation as the targets (Small-Animal-NPs and Large-Object-NPs) $d^{\prime}=.39$, $t(23)=3.00, p<.01 .^{4}$ Mean $d^{\prime}$-values for each participant were subjected to a one-way ANOVA with the factor Prime Type (TP, Same-Cov-NP, and primes with a different co-variation as the targets, i.e. Small-Animal-NP and Large-Object-NP). It revealed that Prime

\footnotetext{
${ }^{4}$ Computing $d^{\prime}$ separately for Small-Animal-NPs and Large-Object-NPs was not possible because prime pictures requiring a small response were treated as signal, whereas prime pictures requiring a large response were considered as noise. In order to assess discrimination performance for these prime types separately, percent of correct answers was computed whereby a performance of $50 \%$ indicates the chance level of guessing. Discrimination performance in percent was $58.3 \%, t(23)=2.6, p<.05$, for SmallAnimal-NPs and $56,2 \%, t(23)=1.9, p>.59$, for Large-Object-NPs
} 
Type had a significant effect on prime visibility $d^{\prime}, F(2,46)=18.35$, $p<.001, \eta^{2}=.44$, MSE $=10.5$.

To test whether the congruency effect is related to prime visibility, a priming index for the RT congruency effect was computed for each participant and each prime type. A subsequently performed linear regression analysis revealed no significant correlation between the three individual $d^{\prime}$-values (for Small-Animal-NP and Large-Object-NP the same $d^{\prime}$-value was used) and the priming index for TPs with $r=-.190, F(1,23)=.82, p>.37$, for Same-Cov-NPs with $r=.251, F(1,23)=1.48, p>.23$ and for Small-Animal-NPs with $r=.260, F(1,23)=1.59, p>.22$. For Large-Object-NP, the regression was significantly different from zero with $r=-.437, F(1,23)=5.19, p<.05$. However, this was due to a negative correlation. That means an increase in visibility was associated with a decrease of the priming effect. The intercept of the regression was larger than zero for TPs (intercept $=8.74, t(23)=3.71, p<.01$ ), for Same-CovNPs (intercept $=3.99, t(23)=2.98, p<.01$ ), for SmallAnimal-NPs (intercept $=-8.07, t(23)=-5.65, p<.001)$ and for Large-Object-NP (intercept $=5.83, t(23)=2.65, p<.05$ ), indicating that significant congruency effects for TPs, SameCov-NPs, and Small-Animal-NPs (inverted effect) are associated with $d^{\prime}$-values of zero.

\section{Discussion}

In Experiment 3, prime pictures presented as targets (TPs) as well as prime pictures never presented as targets but with the same covarying feature as targets, i.e. small objects and large animals (Same-Cov-NP), elicited congruency effects. More importantly however, a reversed congruency effect was observed for primes depicting small animals (Small-Animal-NP). This indicates that prime pictures of small animals did not induce priming regarding the category "small," but regarding the category "large" that covaried with the category animal for the targets. There was no congruency effect for prime pictures of large objects (LargeObject-NPs). To summarize, as in Experiment 2, we found priming from novel primes suggesting late selection. Interestingly, the direction of the congruency effect was influenced by the second feature of the primes, that is, their belonging to the category animals. This implies that the conceptual identity of the prime pictures as animal or object had been processed.

Regarding the reversed congruency effect, the question arises whether participants just redrafted the small-large categorization into an animal-object categorization or if small-large congruence still had an impact on priming. In order to find out whether the congruency effect of animal-object congruence and small-large congruence is additive or if solely animal-object congruence has elicited priming, a further analysis was conducted, concentrating exclusively on novel primes. For this analysis, mean RTs for correct responses were submitted to a three-way ANOVA with the within-subject factors Target Type (small object pictures and large animal pictures), Size Congruency (congruent when prime and target are both either small or large and incongruent when either prime or target is small whereas the other is large), and Category Congruency (congruent when prime and target are both animals or both objects and incongruent when either prime or target is an animal, whereas the other is an object). In this analysis, a significant effect of the factor Category Congruency would indicate that novel prime pictures' membership to the category animal or object had an impact on priming, whereas significance of the factor Size Congruency would indicate that novel prime pictures are still analyzed according to the task instruction. The factor Target Type was significant, $F(1,23)=15.4, p<.001, \eta^{2}=.40, M S E=$ $25,296.9$, as well as the factor Category Congruency, $F(1,23)=$ $34.1, p<.001, \eta^{2}=.60, M S E=10,555.0$. Neither the factor Size Congruency reached significance, $F(1,23)=2.72, p>.11, \eta^{2}=$ .11 , nor any of the interactions $p s>.12^{5}$. Participants responded faster to targets depicting large animals (424 ms) than small objects (447 ms). They also responded faster when primes' and targets' categories were congruent (429 ms) rather than incongruent (443 ms). Overall, Size Congruency had a smaller impact than Category Congruency. Taken together, this further analysis implies that at least some participants had just redrafted their task from the small-large categorization in an object-animal categorization because Category Congruency but not Size Congruency was decisive for congruency effects. Debriefing participants corroborates this inference. Twenty of the 24 participants reported that they had realized that all targets picturing objects were small and all targets picturing animals were large. Moreover, 12 participants stated that they had intentionally redrafted their task from smalllarge to animal-object or to animal-not animal. Consequently, the physical size of the depicted animals/objects became task irrelevant. Furthermore, it is important to note that none of the participants realized that there were prime pictures of small animals and large objects because realizing these primes would have rendered the prevailing response strategy insufficient (realizing that for example small animals afford a different response than large animals).

We conjecture that participants redrafted the task from a smalllarge discrimination in an animal-object classification because this enables faster responding. In Experiment 3, participants responded in average $36 \mathrm{~ms}$ faster and made $.4 \%$ less errors than in Experiment 2. For the small-large categorization in Experiment 2, the size of the depicted animals had to be considered and compared with the reference object. By contrast, in Experiment 3, redrafting the task in an animal-object classification enabled participants to derive the appropriate response from the mere identity (on the basic levels animal or object) of the target picture resulting in faster responses.

Next, the question arises why there was a reversed congruency effect for prime pictures of small animals but no congruency effect at all for prime pictures of large objects. In addition, small pictures and large objects differed when presented as targets because participants responded faster ( $23 \mathrm{~ms}$ in the analysis above) to targets

\footnotetext{
${ }^{5}$ The same ANOVA was computed on error rates. The factor Category Congruency was significant, $F(1,23)=7.5, p<.05, \eta^{2}=.25$, MSE $=$ .017 , indicating that participants made more errors when prime and target category were incongruent $(7.3 \%)$ rather than congruent $(5.4 \%)$. The factor Target Type $F(1,23)=3.4, p<.08, \eta^{2}=.13, M S E=.018$, as well as the factor Size Congruency $F(1,23)=3.5, p<.08, \eta^{2}=.13, M S E=.005$ were marginally significant, indicating that participants made more errors responding to target pictures of small objects $(7.3 \%)$ rather than of large animals $(5.4 \%)$ and that participants made more errors when prime and target size were incongruent $(6.9 \%)$ rather than congruent $(5.8 \%)$. None of the interactions was significant, $p \mathrm{~s}>.23$. Thus, concerning error rates it seems that size congruency matters somewhat.
} 
picturing large animals than to targets picturing small objects. We conjecture that animals form a more coherent category than objects. Animals in general do not seem to afford very different behavioral responses at least for the average nonfarmer member of a western society. Therefore, priming might extend more easily to novel animal primes regardless of their size. In contrast, a large object like, for example, a house has not very much in common with small objects like a trumpet or a fork, and it is linked to quite different behavioral contexts. Moreover, even the small objects like a trumpet, a hammer, or a fork do not form a coherent category itself but they fit better to subcategories like for example music instruments, tools, and cutlery. Alternatively, the large objects differ more regarding their possible size (there are small and large houses or vehicles, etc.), whereas the pictured animals do not differ much regarding their real physical size. Because the objects can be associated with large and small at the same time (despite that they are all clearly larger than the size reference), they may not be able to activate one of the categories as automatic as the animal primes $^{6}$. Thus, transfer of priming to novel primes is more difficult-be it for primes picturing small objects as well as large objects. Indeed a differential analyses of the congruency effects of Same-Cov-NPs showed that only primes picturing large animals elicited a congruency effect of $49 \mathrm{~ms}, t(23)=6.05, p<.001$, whereas the congruency effect of primes picturing small objects was $-5 \mathrm{~ms}$ and not significant, $t(23)=-.62, p>.54$. As a consequence, when redrafting the task in an animal-object classification, priming transferred to novel animal pictures but not to novel object pictures.

Finally, again discrimination performance for primes picturing targets (TPs) in Experiment 3 was higher than discrimination performance for the other prime types and discrimination performance for primes that were never presented as targets but were also large animals or small objects (Same-Cov-NPs) was somewhat higher than discrimination performance for primes with a reversed co-variation (Small-Animal-NPs und Large-Object-NPs). This is in line with the assumption that prevailing intentions lead to a better discrimination performance of masked primes.

\section{Experiment 4}

In Experiment 1, primes in a slightly different illustration (DiffIllu-TPs) with the same left-right orientation as the targets elicited priming, whereas primes with the opposite left-right orientation were ineffective. Thus, we argue that with a small target set, perceptual match between prime and target stimuli is a prerequisite for congruency effects in line with the assumption of early selection.

In Experiment 2 and 3, 40 different pictures were presented as targets, respectively. It is possible that these large target sets unintentionally entailed more feature overlap with the novel primes than did the small target set of Experiment 1. If this were the case, priming observed in Experiment 2 and 3 would need an early selection interpretation. In addition, such an accidental change of the amount of feature overlap could be an alternative reason why we found that small animal pictures facilitated the response of large animal pictures in Experiment 3 while small object pictures did not influence responding to large object pictures.

Experiment 4 was designed as a more specific control in order to test directly the influence of feature overlap when using picture stimuli as primes and targets. Participants were instructed to categorize pictures of animals and objects as smaller or larger than the reference object. Again 40 different target pictures were used, but now 10 pictures showed large objects, 10 showed large animals, 10 showed small objects, and 10 showed small animals. Thus, pictures that share many perceptual features (e.g. a small animal and a large animal) demand different responses, whereas pictures that do not share perceptual features (e.g. a small animal and a small object), demand the same size classification.

This design allows us to asses and to compare the influence of feature overlap on the amount of observed congruency effects. If feature overlap had contaminated our former results, we would expect to find no priming if primes and targets belong to different categories (i.e. small animals should not facilitate responding to small objects). Even a reversed congruency effect would be plausible and indicate that perceptual features influence responding to the target to a stronger degree than congruency according to the instructed size classification (i.e. small animals would facilitate responding to large animals). In contrast, a considerable congruency effect even when prime and target belong to different categories would emphasize that subliminal stimuli were subject to late selection because prime processing would entail information regarding the physical size of the subliminal stimuli for the picture stimuli we used.

\section{Method}

\section{Participants}

Twenty-four students (four males) of the University of Würzburg with an average age of 22.4 years participated in Experiment 4 in fulfillment of course requirements. All reported having normal or corrected-to-normal vision, and were not familiar with the purpose of the experiment. An experimental session lasted approximately $45 \mathrm{~min}$.

\section{Apparatus and Stimuli}

Apparatus and stimuli were similar to Experiment 3 except for the following changes: Again, we presented a large set of targets consisting of 10 pictures of small animals (bee, squirrel, bird, grasshopper, fish, mouse, caterpillar, ant, snail and duck), 10 pictures of small objects (pen, glasses, paper clip, cup, pliers, key, whistle, wristwatch, clothespin and screw), 10 pictures of large objects (bed, couch, aircraft, fence, baby carriage, truck, commode, piano, house and chair), and 10 pictures of large animals (donkey, horse, alligator, leopard, cow, kangaroo, lion, rhinoceros, deer and camel). Sixteen pictures were used as primes: 4 pictures of small animals (grasshopper, ant, mouse and bird), 4 pictures of small objects (pliers, key, whistle and pen), 4 pictures of large animals (rhinoceros, donkey, camel and lion), and 4 pictures of large objects (chair, bed, aircraft and piano). All primes were also presented as targets. All pictures were greyscale shaded images and appeared in a white rectangle $2 \mathrm{~cm}$ high by $3 \mathrm{~cm}$ wide (seven target pictures were $3 \mathrm{~cm}$ high by $2 \mathrm{~cm}$ wide). Each prime-target-

\footnotetext{
${ }^{6}$ We thank an anonymous reviewer for mentioning this alternative.
} 
pair was presented once in the experiment resulting in 640 trials (16 primes $\times 40$ targets).

\section{Design and Procedure}

Design and procedure was analogues to Experiment 1. Participants were instructed to categorize the depicted animals and objects as being smaller or larger than the reference object.

\section{Results}

\section{Congruency Effect}

Trials with RTs deviating more than 2.5 SDs from the mean RT of each participant and each condition were excluded. Mean RTs and PEs were computed for each participant for each combination of the factors Category Congruency (congruent when prime and target are both animals or both objects and incongruent when either prime or target is an animal whereas the other is an object, that means congruent reflects high feature overlap while incongruent reflects low overlap) and Size Congruency (congruent and incongruent). Averaged data across all participants are presented in Figure 5.

Mean RTs for correct responses were submitted to an ANOVA with the within-subject factors Category Congruency and Size Congruency. Only the factor Size Congruency was significant, $F(1,23)=71.3, p<.001, \eta^{2}=.76, M S E=11,733.1$. Neither the factor Category Congruency, $p>.25$, nor the interaction Category Congruency $\times$ Size Congruency, $p>.11$, reached significance. Participants responded faster for size-congruent $(430 \mathrm{~ms})$ than size-incongruent $(452 \mathrm{~ms})$ primes irrespective of high or low feature overlap (size-congruency effects amounted to $25 \mathrm{~ms}$ vs. 19 $\mathrm{ms}$ for category-congruent vs. category-incongruent prime-target pairs.).

The overall mean error rate was $7.6 \%$. The pattern of the error rates mirrors the RT data. The same ANOVA on errors revealed a significant effect only for the factor Size Congruency $F(1,23)=$ 47.4, $p<.001, \eta^{2}=.67, M S E=.057$. Neither the factor Category Congruency, $p>.74$, nor the interaction Category Congruency $\times$ Size Congruency, $p>.53$, reached significance. Participants made more errors in size-congruent than in size-incongruent trials irrespective of high or low feature overlap (size-congruency effects of $5.1 \%$ vs. $4.6 \%$ for category-congruent vs. category-incongruent prime-target pairs).

\section{Prime Visibility}

Overall discrimination for prime pictures was $d^{\prime}=.51$ (the mean hit rate was $61.9 \%$, false alarm rate $42.4 \%$ ) and deviated from zero, $t(23)=6.0, p<.001$. To test whether the congruency effect is related to prime visibility, a priming index for the RT congruency effect was computed for each participant as in Experiment 1. A subsequently performed linear regression analysis revealed no significant correlation between the individual $d^{\prime}$ value and the priming index with $r=.091, F(1,23)=.18$, $p>$.67. The intercept of the regression was larger than zero (intercept $=5.61, t(23)=5.3, p<.001$ ), indicating that significant congruency effects are associated with $d^{\prime}$-values of
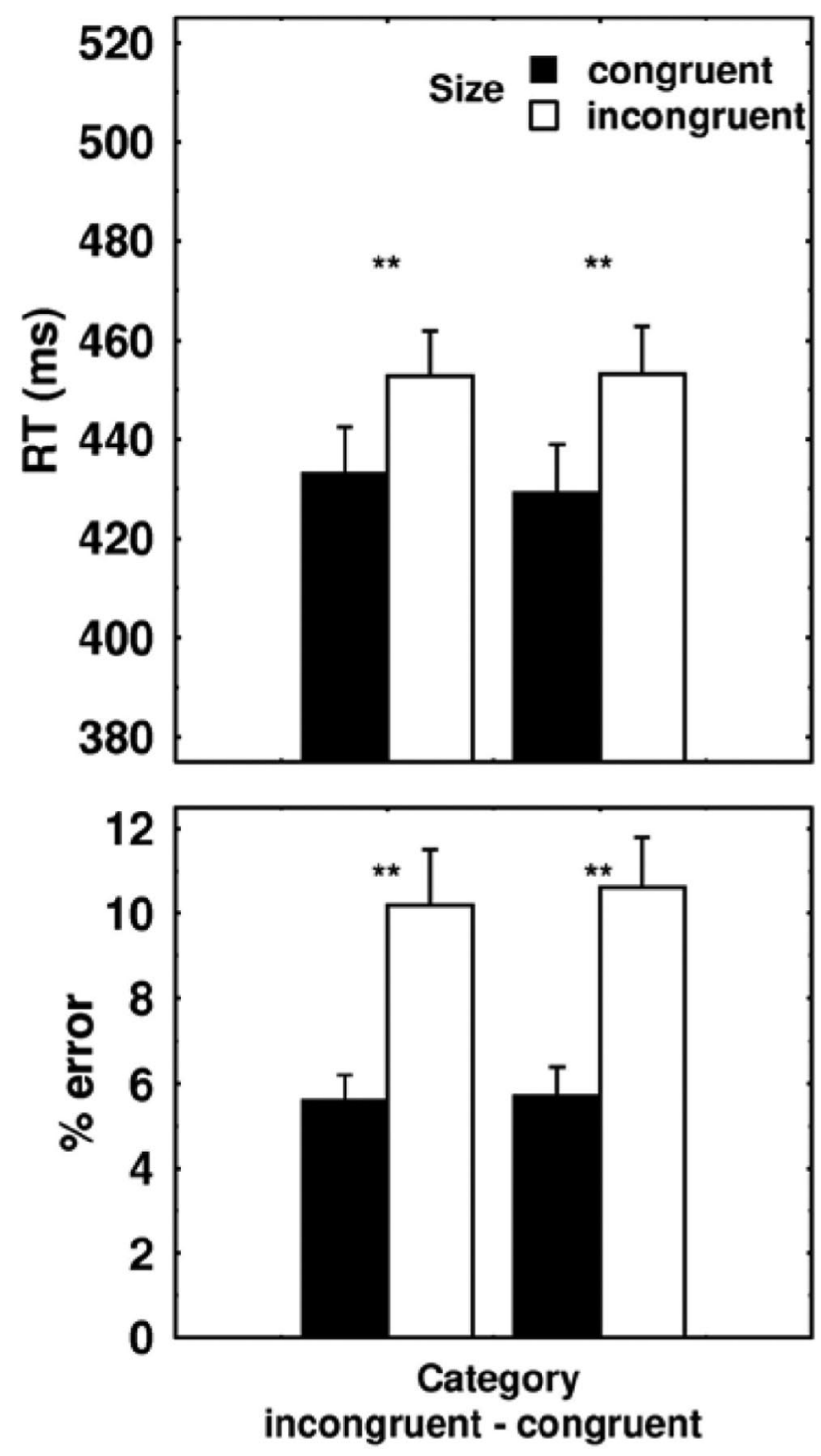

Figure 5. Mean response times (RTs; upper panel) and error rates (lower panel) depending on Category Congruency and Size Congruency in Experiment 4 . Lines represent standard errors. Asterisks indicate the significance level: ${ }^{*} p<.05 .{ }^{* *} p<.01$.

zero. Thus, the observed congruency effect is independent on individual prime visibility.

\section{Discussion}

Participants responded significantly slower in incongruent compared to congruent trials irrespective of whether primes and targets belonged to the same (high feature overlap) or different categories (low feature overlap). Descriptively, there was a somewhat higher congruency effect for trials with high feature overlap (25 ms; 5.1\% errors) compared to trials with low feature overlap (19 ms; $4.6 \%$ errors).

Please note that regarding feature overlap between primes and targets, two forms can be discriminated, which both accord with an 
early selection account: First, overlap between primes and potential targets (i.e., the target set) and second, overlap between prime and target within a trial. There is evidence that both forms of feature overlap can take effect under appropriate conditions. For example, the former form of feature overlap is indicated by paradoxical priming effects. These effects can occur when primes share more perceptual features with potential targets of a primeunrelated semantic response category than with potential targets of a prime-related semantic category (Abrams \& Greenwald, 2000; Greenwald et al. 2003). There is also evidence for the latter, trialwise, impact of feature overlap. For example, when Arabic numerals as well as number words are used as prime and target stimuli, then responses are faster when targets are preceded by primes with the same compared to a different notation (e.g. Van den Bussche, Segers, \& Reynvoet, 2008). Bodner and Dypvik (2005) showed an even more extreme form of feature overlap between prime and target. They demonstrated that participants respond faster if prime and target were identical (1-1, 2-2, etc.) compared to congruent $(1-3,2-4$, etc.) in a parity judgment task. Consequently, not just response meaning but also perceptual feature overlap within a trial speeds up responding. Experiment 4 shows that both forms of a potential contamination through overlapping features are unlikely in our previous experiments. First, if feature overlap between prime and the potential target set would cause congruency effects, we would not expect any congruency effects in Experiment 4 because for example a small animal shares roughly the same amount of features with small and large animals. Consequently, a small animal prime should similarly activate the small as well as the large response thereby nullifying response-related congruency effects. Second, if feature overlap within a trial were responsible for congruency effects, we should find a strong impact of category congruency instead of, or in addition to, an impact of size congruency. Obviously this was not the case.

Concerning our Experiments 1-3, we can therefore conclude that feature overlap between primes and the potential target sets and primes and targets within a trial contributes if at all only slightly to the observed congruency effects. Much more decisive for the impact of primes are the (self-) instructed task and the demanded response assignments. By using a slightly different experimental procedure (the so called response window technique), Klinger, Burton, and Pitts (2000) derived a very similar conclusion, based on the observation, that the assignment of prime and target to identical responses did facilitate responding whereas affective, semantic or animacy similarity of prime and target did not.

Contrary to these observations, a recent study by Reynvoet, Gevers, and Caessens (2005; see also Klauer, Musch, \& Eder, 2005 , for a similar result) found a significant effect of category congruency. When using alphanumeric stimuli from two different categories (letters and digits) and asking participants to perform two classification tasks (vowel vs. consonant classification and smaller or larger categorization) in a subliminal priming experiment, Reynvoet et al. showed that category match facilitated responding to the target. Participants responded faster if prime and target belonged to the same category than if they belonged to different categories. An explanation for the different results of Experiment 4 and the study of Reynvoet and colleagues (2005) may lie in different experimental tasks. Whereas Reynvoet and colleagues administered two different tasks for the two stimulus categories (see also Kiesel, Kunde, \& Hoffmann, 2007a; Klauer et al., 2005), our participants always performed the same task on each stimulus irrespective of the category of the stimulus. Probably, the additional requirement to switch tasks led to the advantage for same category prime-target pairs. Alternatively, the picture stimuli used in the current study may have prevented an effect of category congruency that was observed for digits and letters (Kiesel et al., 2007a; Reynvoet et al., 2005) or word stimuli (Klauer et al., 2005).

\section{General Discussion}

The present study examined under which conditions masked stimuli are subjected to early or late selection. Therefore, we manipulated the applied set of target stimuli by varying the amount and the semantic affiliation of the presented target pictures. Using pictures as primes and targets we found both, early selection in Experiment 1 as well as late selection in Experiment 2 and 3 for identical conditions of prime presentation. Experiment 1 showed that with a small target set, participants differentiate stimuli on a concrete perceptual level. With only four pictures as targets only the very same prime pictures were selected and induced congruency effects. A slight change of the outward appearance, like changing the left-right orientation, of the depicted prime pictures was sufficient to reduce priming - thus, primes were selected early based on their sensory resemblance to targets. In Experiment 2, 40 different targets were used and consequently targets had to be differentiated on the basis of semantic features. Congruency effects spread to novel prime pictures from the same category as the targets (animals) while prime pictures depicting objects remained ineffective despite that they could have been easily classified as small or large. Thus, even with a large set of targets, priming did not transfer to each appropriate prime stimulus but masked stimuli were selected rather late depending on their membership to the category animal. Experiment 3 investigated masked priming while a second feature co-varied with the required response and allowed redrafting the task from the small-large categorization into an easier animal-object categorization. Redrafting of the task led to a reversed congruency effect with respect to the instructed task. That is, prime pictures of small animals did not induce priming regarding the category small, but regarding the category large, which co-varied with animal pictures presented as targets. Finally, Experiment 4 was designed to find out, whether overlap of perceptual features had an impact on priming. The same response was required for the pictures of small animals and small objects (or large animals and large objects), whereas pictures of small animals and large animals (or small objects and large objects) demanded the other response. Now, we observed congruency effects according the instructed task in trials with many overlapping features between prime and target as well as in trials with no overlapping features. For instance, the prime picture of a small animal facilitated not only the response to a target picture of another small animal, but also to a target picture of a big object. Feature overlap had no significant additional impact. Thus, the stimulus material used in Experiment 2 and 3 led indeed to a late selection-not to a perceptual driven early selection. 


\section{Early and Late Selection for Subliminal Stimuli}

Concerning the processing of masked stimuli there has been a controversy similar to the discussion whether supraliminal stimuli are subjected to early or late selection. Please note, that within this regard the rather old fashioned term "early vs. late selection" does not necessary imply that processing takes place at specific points in time (indeed our study does not tell anything about the time of selection). Rather, we use the term to refer to selection criteria, that is, early processing refers to physical selection criteria and late processing to semantic selection criteria. While there is a wide agreement that unconscious stimuli are processed, the mechanisms underlying masked priming are controversially debated. Especially, the question how deep subliminally presented primes are processed bothers the researchers: Are subliminal primes processed semantically (e.g. Dehaene et al., 1998; Reynvoet et al., 2005)? Or is the acquisition of stimulus-response links a prerequisite for subliminal primes to activate motor responses (e.g., Damian, 2001)?

On the one hand, processing of unconscious stimuli is considered to be dumb and without involvement of higher cognitive functions; i.e., it is considered to be Zombie-like (Tapia \& Breitmeyer, 2006; Cleeremans \& Jiménez, 2002). This view reflects the assumption that stimulus processing is subject to early selection (Cherry, 1953; Broadbent, 1958). Unattended stimuli are just processed according to their sensory, physical, or superficial features. Consequently, masked primes elicit congruency effects only if there are direct links for these stimuli and the required responses. These $\mathrm{S}-\mathrm{R}$ links have been acquired in the course of the experiment when participants responded to identical stimuli presented as targets. Indeed some experiments (Abrams \& Greenwald, 2000; Damian, 2001; Greenwald et al., 2003) demonstrated that novel primes were ineffective and priming was restricted to target primes or even parts of them. For example, Damian (2001) presented 12 words denoting objects as targets and the task was to categorize them as small or large. Prime words affected performance only when they were used as targets as well. Moreover, the impact of these words, i.e. the congruency effect increased in the course of the experiment indicating that the size of the congruency effect depended on the strength of acquired S-R links. In contrast, novel prime words failed to elicit congruency effects and consequently, building up S-R links through conscious responding to specific stimuli was considered as a prerequisite for subliminal stimuli to induce priming.

On the other hand, there are accounts assuming that there is no difference between processing of visible and invisible stimuli. Cleeremans and Jiménez (2002) caricatured this position of sophisticated unconscious processing tellingly with a "Commander Data" theory. This assumption is in line with the late selection account (Deutsch \& Deutsch, 1963). Masked primes influence target processing through their semantic meaning. Thus, subliminal priming occurs because participants apply task instructions on any suitable stimulus, irrespective whether this stimulus is consciously perceived or not (Dehaene et al., 1998; Reynvoet et al., 2005). Experiments demonstrating priming from novel, unseen prime stimuli have been considered as evidence for this view (e.g. Dell'Acqua \& Grainger, 1999; Naccache \& Dehaene, 2001). At a first sight the finding that priming is restricted to target primes when using a small target set seems to contradict findings by Naccache and Dehaene (2001). Consider that in their experiment, participants had to judge whether digits are smaller or larger than 5 , and in the experiments targets were the numbers $1,4,6$, and 9. In these conditions the numbers 1 and 4 become selected for the left response and the numbers 6 and 9 become selected for the right response. All we know about the mental representation of numbers tells us that they form an intimately integrated representation that is often described as a mental number line (Galton, 1880; Göbel, Walsh, \& Rushworth, 2001). If now the numbers 1 and 4 have become selected for a left response, it seems likely that the mentally enclosed numbers 2 and 3 might similarly become selected when there is no obvious harm in doing so. If this "incidental recollection" account is correct, it should be less likely that unseen stimuli become considered as potential targets when the semantic distance to the experienced targets is larger. This seems to be so. When the digits 3 and 4 are used as targets (and selected as response criteria) the neighboring, but not enclosed, numbers 1 and 2 exert essentially no priming effect (Kunde et al., 2003, Exp. 2).

Further, the current results of Experiment 2 and 3 might be considered as a successful replication of the Dell'Acqua and Grainger (1999) study. Novel picture stimuli elicited congruency effects when presented as masked primes. Interestingly, Dell'Acqua and Grainger (1999) observed congruency effects by picture stimuli even while using words as target stimuli. We conjecture that pictures are subject to late selection (even beyond the expected target format) when broad target categories are used. Such an assumption is in line with recent neurophysiological data (Pulvermüller, 2001; Vandenberghe, Price, Wise, Josephs, \& Frackowiak, 1996) that suggest that words denoting concrete objects activate similar cortical networks as images of the same objects.

Yet, altogether, neither the account of "Zombie-like" restricted prime processing nor the account of "Commander Data-like" semantic prime processing alone can account for all the available evidence. The present experiments underline that these apparently contradictory positions need not necessarily be incommensurate. Whereas separate studies showed that masked primes are subject to early selection (Damian, 2001; Abrams \& Greenwald, 2000; Greenwald et al., 2003) or late selection (Dehaene et al., 1998; Naccache \& Dehaene, 2001; Reynvoet et al., 2005), we demonstrated in one study that both is possible. Essentially the same masked primes affected responding either through perceptual features or through conceptual meaning depending on concrete experimental conditions. By varying the number and the semantic affiliation of the perceived target pictures (Exp. 1 and 2) and by creating a task that allows an alternative classification (Exp. 3) we triggered early as well as late selection. Thus, unconscious stimuli can bias behavior by means of both perceptual and semantic features, according to the prestimulus expectations and selection criteria imposed by visible targets.

In addition, our result may be taken as evidence that distinguishing between early selection, on the one hand, and late selection on the other hand is a crude simplification when characterizing stimulus processing. At least for consciously presented stimuli, processing does not occur in strictly unidirectional serial stages, but bottom-up and top-down processes interact (e.g., Hommel, 2000). The current results clearly reveal that processing of consciously presented targets influences processing of unconscious stimuli. Thus, there is a top-down impact caused by conscious stimuli on unconscious stimulus processing. Hereby, the dichotomy in early and late selection seems to be obsolete. Yet, it is currently under debate whether unconsciously presented stimuli similarly impact on top-down processes. For 
example, the global neural workspace theory (Baars, 1988; Dehaene \& Naccache, 2001) as well as the action trigger account (Kiesel, Kunde, \& Hoffmann, 2007b; Kunde, Kiesel, \& Hoffmann, 2003) assume that unconsciously presented stimuli do not themselves modify processing whereas processing of unconsciously presented stimuli is heavily influenced by topdown control settings (for similar suggestions see Lamme, 2003; Lamme \& Roelfsema, 2000). Evidence for such an "asymmetry between top-down effects and unconscious cognition" (Naccache, 2008, p. 1359) was brought forward in several recent studies (e.g. Kiesel, Kunde \& Hoffmann, 2006; Kunde, 2003; Merikle, Joordens, \& Stolz, 1995; Van den Bussche, et al., 2008). For example, Kunde (2003) reported that sequential modulations of prime-target congruency effects only occurred when a response conflict in a preceding trial was induced by a clearly visible prime. In contrast, response conflict induced by an unconsciously presented stimulus did not evoke any sequential modulation effects. Thus, the concept of distinguishing processing in early or late selection can still be applied for unconscious information processing.

\section{Top-Down Processes}

Apart from the assumption that prestimulus expectations determine prime processing, strong evidence is accumulating that other top-down processes influence the processing of masked primes as well. It has been shown that congruency effects depend on attention, task instructions and task context: First, masked priming in a number comparison task is restricted to conditions in which participants allocate their temporal attention to the time window of prime occurrence (Naccache, Blandin, \& Dehaene, 2002). Likewise, Lachter, Forster, and Ruthruff (2004) found that priming depends on spatial attention. Second, varying instructions determined whether prime numbers were analyzed up to their quantity meaning or not (Kunde et al., 2003, Exp. 3). In addition, congruency effects adapted to the currently instructed S-R mappings even when the S-R-mappings were reversed trial by trial (Neumann \& Klotz, 1994, Exp. 4; but see Kiesel et al., 2007a; for priming according to multiple task instructions). Third, the current experiments revealed that the context of the task determines which primes induce congruency effects. Within number comparison tasks, a similar observation has already been reported: When only digits were presented as targets, only prime digits but not numeral words induced congruence effects (Kunde et al., 2003, Exp. 4, for a close discussion see Kunde, Kiesel, \& Hoffmann, 2005; Van Opstal, Reynvoet, \& Verguts, 2005a, 2005b).

To conclude, considering the impact of top-down influences may render the controversy whether the impact of masked primes is due to acquired $\mathrm{S}-\mathrm{R}$ connections or semantic relations between primes and targets superfluous-similarly to the former controversy whether attention for supraliminal stimuli is accompanied by early or late selection processes.

\section{References}

Abrams, R. L., \& Greenwald, A. G. (2000). Parts outweigh the whole (word) in unconscious analysis of meaning. Psychological Science, 11, $118-124$.

Abrams, R. L., Klinger, M. R., \& Greenwald, A. G. (2002). Subliminal words activate semantic categories (not automated motor responses). Psychonomic Bulletin \& Review, 9, 100-106.

Allport, A. (1987). Selection for action: Some behavioial and neurophysiological considerations of attention and action. In H. Heuer \& A. F. Sanders (Eds.), Perspectives on perception and action (pp. 395-420). Hillsdale, NJ: Erlbaum.

Ansorge, U., \& Neumann, O. (2005). Intentions determine the effect of invisible metacontrast-masked primes: Evidence for top-down contingencies in a pheripheral cuing task. Journal of Experimental Psychology: Human Perception and Performance, 31, 762-777.

Baars, B. J. (1988). A cognitive theory of consciousness. Cambridge: Cambridge University Press.

Bates, E., D’Amico, S., Jacobsen, T., Szekely, A., Andonova, E., Devescovi, A., et al. (2003). Timed picture naming in seven languages. Psychonomic Bulletin \& Review, 10, 344-380.

Bodner, G. E., \& Dypvik, A. T. (2005). Masked priming of number judgments depends on prime validity and task. Memory \& Cognition, 33, $29-47$.

Boyer, J., \& Ro, T. (2007). Attention attenuates metacontrast masking. Cognition, 104, 135-149.

Broadbent, D. E. (1958). Perception and communication. In Perception and communication. Oxford: Pergamon Press.

Cherry, E. C. (1953). Some experiments on the recognition of speech with one and with two ears. Journal of the Acoustic Society of America, 25, 975-979.

Cleeremans, A., \& Jiménez, L. (2002). Implicit learning and consciousness: A graded, dynamic perspective. In R. M. Frensch \& A. Cleeremans (Eds.), Implicit learning and consciousness (pp. 1-40). Hove, UK: Psychology Press.

Corteen, R. S., \& Dunn, D. (1974). Shock associated words in a nonattended message. A test for momentary awareness. Journal of Experimental Psychology, 102, 1143-1144.

Corteen, R. S., \& Wood, B. (1972). Automatic responses to shock associated words in an unattended channel. Journal of Experimental Psychology, 94, 308-313.

Damian, M. F. (2001). Congruity effects evoked by subliminally presented primes: Automaticity rather than semantic processing. Journal of Experimental Psychology: Human Perception and Performance, 27, 154-165.

Dawson, M. E., \& Schell, A. M. (1982). Electrodermal responses to attended and nonattended significant stimuli during dichotic listening. Journal of Experimental Psychology: Human Perception and Performance, 8, 315-324.

Dehaene, S., \& Naccache, L. (2001). Towards a cognitive neuroscience of consciousness: Basic evidence and a workspace framework. Cognition, 79, 1-37.

Dehaene, S., Naccache, L., Le Clec'H, G., Koechlin, E., Mueller, M., Dehaene-Lambertz, G., et al. (1998). Imaging unconscious semantic priming. Nature, 395, 597-600.

Dell'Acqua, R., \& Grainger, J. (1999). Unconscious semantic priming from pictures. Cognition, 73, B1-B15.

Deutsch, J. A., \& Deutsch, D. (1963). Attention: Some theoretical considerations. Psychological Review, 70, 80-90.

Draine, S. C., \& Greenwald, A. G. (1998). Replicable unconscious semantic priming. Journal of Experimental Psychology: General, 127, 286-303.

Egeth, H. E. (1977). Attention and preattention. In G. H. Bower (Ed.), The psychology of learning and motivation (Vol. 7). New York: Academic Press.

Enns, J. T., \& Oriet, C. (2007). Visual Similarity in masking and Priming: The critical role of task relevance. Advances in Cognitive Psychology, 3, 211-226.

Farah, M. J. (1994). Category-specificity in object recognition: Clues from prosopagnosia and alexia. In M. J. Farah and G. Ratcliff (Eds.), The neuropsychology of high-level vision: Collected tutorial essays. Hillsdale, NJ: Erlbaum Associates. 
Galton, F. (1880). Visualised numerals. Nature, 21, 252-256.

Göbel, S., Walsh, V., \& Rushworth, M. F. S. (2001). The mental number line and the human angular gyrus. NeuroImage, 14, 1278-1289.

Greenwald, A. G., Abrams, R. L., Naccache, L., \& Dehaene, S. (2003). Long-term semantic memory versus contextual memory in unconscious number processing. Journal of Experimental Psychology: Learning, Memory, and Cognition, 29, 235-247.

Greenwald, A. G., Draine, S. C., \& Abrams, R. L. (1996). Three cognitive markers of unconscious semantic activation. Science, 273, 1699-1702.

Greenwald, A. G., Klinger, M. R., \& Schuh, E. S. (1995). Activation by marginally perceptible ("subliminal") stimuli: Dissociation of unconscious from conscious cognition. Journal of Experimental Psychology: General, 124, 22-42.

Hautus, M. J. (1995). Corections for extreme proportions and their biasing effets on estimated values of $d^{\prime}$. Behavior Research Methods, Instruments, and Computers, 27, 289-297.

Holender, D. (1986). Semantic activation without conscious identification in dichotic listening, parafoveal vision, and visual masking: A survey and appraisal. The behavioral and brain sciences, 9, 1-66.

Hommel, B. (2000). The prepared reflex: Automaticity and control in stimulus-response translation. In S. Monsell \& J. Driver (Eds.), Control of cognitive processes: Attention and performance XVIII (pp. 247-273). Cambridge, MA: MIT Press.

Kiefer, M. (2002). The N400 is modulated by unconsciously perceived masked words: Further evidence for an automatic spreading activation account of N400 priming effects. Cognitive Brain Research, 13, 27-39.

Kiefer, M. (2007). Top-down modulation of unconscious 'automatic' processes: A gating framework. Advances in Cognitive Psychology, 3, 289-306

Kiefer, M., \& Spitzer, M. (2000). Time course of conscious and unconscious semantic brain activation. NeuroReport, 11, 2401-2407.

Kiesel, A., Kunde, W., \& Hoffmann, J. (2006). Evidence for task-specific resolution of response conflict. Psychonomic Bulletin and Review, 13, $800-806$

Kiesel, A., Kunde, W., \& Hoffmann, J. (2007a). Unconscious priming according to multiple S-R rules. Cognition, 104, 89-105.

Kiesel, A., Kunde, W., \& Hoffmann, J. (2007b). Mechanisms of subliminal response priming. Advances in Cognitive Psychology, 3, 307-315.

Kiesel, A., Kunde, W., Pohl, C., \& Hoffmann, J. (2006). Priming from novel masked stimuli depends on target set size. Advances in Experimental Psychology, 1, 9-17.

Kiesel, A., Wagener, A., Kunde, W., Hoffmann, J., Fallgatter, A. J., \& Stöcker, C. (2006). Unconscious manipulation of free choice in humans. Consciousness \& Cognition, 15, 397-408.

Klauer, K. C., Eder, A. B., Greenwald, A. G., \& Abrams, R. L. (2006). Priming of semantic classifications by novel subliminal prime words. Consciousness and Cognition, 16, 63-83.

Klauer, K. C., Musch, J., \& Eder, A. B. (2005). Priming of semantic classifications: Late and response-related, or earlier and more central? Psychonomic Bulletin and Review, 12, 897-903.

Klinger, M. R., Burton, P. C., \& Pitts, G. S. (2000). Mechanisms of Unconscious Priming I: Response Competition Not Spreading Activation. Journal of Experimental Psychology: Learning, Memory, and Cognition, 26, 441-455.

Kunde, W. (2003). Sequential modulations of stimulus-response correspondence effects depend on awareness of response conflict. Psychonomic Bulletin \& Review, 10, 198-205.

Kunde, W., Kiesel, A., \& Hoffmann, J. (2003). Conscious control over the content of unconscious cognition. Cognition, 88, 223-242.

Kunde, W., Kiesel, A., \& Hoffmann, J. (2005). On the masking and disclosure of unconscious elaborate processing: A reply to Van Opstal, Reynvoet, \& Verguts. Cognition, 97, 99-105.

Lachter, J., Forster, K., \& Ruthruff, E. (2004). Forty-five years after
Broadbent (1958): Still no identification without attention. Psychological Review, 111, 880-913.

Lamme, V. A. F. (2003). Why visual attention and awareness are different. Trends in Cognitive Sciences, 7, 12-18.

Lamme, V. A. F., \& Roelfsema, P. R. (2000). The distinct modes of vision offered by feedforward and recurrent processing. Trends in Neuroscience, 23, 571-579.

Mack, A., \& Rock, I. (1998). Inattentional blindness. Cambridge, MA: MIT Press.

Marcel, A. (1983). Conscious and unconscious perception: An approach to the relations between phenomenal experience and perceptual processes. Cognitive Psychology, 15, 238-300.

Merikle, P. M., Joordens, S., \& Stolz, J. A. (1995). Measuring the relative magnitude of unconscious influences. Consciousness \& Cognition, 4, 422-439.

Moray, N. (1959). Attention and dichotic listening. Quarterly Journal of Experimental Psychology, 11, 56-60.

Müller, H. J., \& Krummenacher, J. (2002). Aufmerksamkeit. In J. Müsseler \& W. Prinz (Eds.), Allgemeine psychologie (pp. 119-177). Heidelberg: Spektrum Akademischer Verlag.

Naccache, L. (2008). Conscious influences on subliminal cognition exist and are asymmetrical: Validation of a double prediction. Consciousness \& Cognition, 17, 1359-1360.

Naccache, L., Blandin, E., \& Dehaene, S. (2002). Unconscious masked priming depends on temporal attention. Psychological Science, 13, 416-424.

Naccache, L., \& Dehaene, S. (2001). Unconscious semantic priming extends to novel unseen stimuli. Cognition, 80, 223-237.

Naccache, L., Gaillard, R., Adam, C., Hasboun, D., Clemenceau, S., Baulac, M., et al. (2005). A direct intracranial record of emotions evoked by subliminal words. Proceedings of the National Academy of Sciences, 102, 7713-7717.

Neumann, O., \& Klotz, W. (1994). Motor responses to nonreportable, masked stimuli: Where is the limit of direct parameter specification? In C. Umilta \& M. Moscovitch (Eds.), Attention and performance XV. Conscious and nonconscious information processing (pp. 123-150). Cambridge: MIT Press

Ostry, D., Moray, N., \& Marks, G. (1976). Attention, practise, and semantic targets. Journal of Experimental Psychology: Human Perception and Performance, 2, 326-366.

Pashler, H. E. (1999). The Psychology of attention. Cambridge: MIT Press.

Pulvermüller, F. (2001). Brain reflections of words and their meaning, Trends in Cognitive Sciences, 5, 517-524.

Reynvoet, B., Gevers, W., \& Caessens, B. (2005). Unconscious primes activate motor codes through semantics. Journal of Experimental Psychology: Learning, Memory, and Cognition, 31, 991-1000.

Rossion, B., \& Pourtois, G. (2004). Revisiting Snodgrass and Vanderwart's object pictorial set: The role of surface detail in basic-level object recognition. Perception, 33, 217-236.

Schlaghecken, F., \& Eimer, M. (2004). Masked prime stimuli can bias "free" choices between response alternatives. Psychonomic Bulletin \& Review, 11, 463-468.

Schneider, W., Eschman, A., \& Zuccolotto, A. (2002). E-prime users' guide. Pittsburgh: Psychology Software Tools Inc.

Tapia, E., \& Breitmeyer, B. G. (2006). Attentional processing capacities of the visual "zombie" and "homunculus." Abstracts of the Psychonomic Society, 47th Annual Meeting, 11, 8.

Treisman, A. (1964). Verbal cues, language, and meaning in selective attention. American Journal of Psychology, 77, 206-219.

Vandenberghe, R., Price, C., Wise, R., Josephs, O., \& Frackowiak, R. S. J. (1996). Functional anatomy of a common semantic system for words and pictures, Nature, 383, 254-256.

Van den Bussche, E., Notebaert, K., \& Reynvoet, B. (2009). Masked primes can be genuinely semantically processed: A picture prime study. Experimental Psychology, 56, 295-300. 
Van den Bussche, E., Segers, G., \& Reynvoet, B. (2008). Conscious and unconscious proportion effects in masked priming. Consciousness \& Cognition, 17, 1345-1358.

Van Opstal, F., Reynvoet, B., \& Verguts, T. (2005a). How to trigger elaborate processing? A comment on Kunde, Kiesel, and Hoffmann (2003). Cognition, 97, 89-97.

Van Opstal, F., Reynvoet, B., \& Verguts, T. (2005b). Unconscious semantic categorization and mask interactions: An elaborate response to Kunde et al. (2005). Cognition, 97, 107-113.
Vorberg, D., Mattler, U., Heinecke, A., Schmidt, T., \& Schwarzbach, J. (2003). Different time courses for visual perception and action priming. Proceedings of the National Academy of Sciences, USA, 100, 62756280.

Received September 24, 2007

Revision received December 21, 2008

Accepted January 12, 2009

\section{Call for Nominations: Emotion}

The Publications and Communications (P\&C) Board of the American Psychological Association has opened nominations for the editorship of the journal Emotion for the years 2012-2017. Elizabeth A. Phelps is the incumbent editor.

Candidates should be members of APA and should be available to start receiving manuscripts in early 2011 to prepare for issues published in 2012. Please note that the P\&C Board encourages participation by members of underrepresented groups in the publication process and would particularly welcome such nominees. Self-nominations are also encouraged. The search is being chaired by Norman Abeles, PhD.

Candidates should be nominated by accessing APA's EditorQuest site on the Web. Using your Web browser, go to http://editorquest.apa.org. On the Home menu on the left, find "Guests." Next, click on the link "Submit a Nomination," enter your nominee's information, and click "Submit."

Prepared statements of one page or less in support of a nominee can also be submitted by e-mail to Emnet Tesfaye, P\&C Board Search Liaison, at emnet@apa.org. 